\title{
An Algebraic Glimpse at Bunched Implications and Separation Logic
}

\author{
Peter Jipsen and Tadeusz Litak
}

Submitted September 2017. Accepted version prepared for publication as of September 27, 2018

\begin{abstract}
We overview the logic of Bunched Implications (BI) and Separation Logic (SL) from a perspective inspired by Hiroakira Ono's algebraic approach to substructural logics. We propose generalized BI algebras (GBI-algebras) as a common framework for algebras arising via "declarative resource reading", intuitionistic generalizations of relation algebras and arrow logics and the distributive Lambek calculus with intuitionistic implication. Apart from existing models of BI (in particular, heap models and effect algebras), we also cover models arising from weakening relations, formal languages or more fine-grained treatment of labelled trees and semistructured data. After briefly discussing the lattice of subvarieties of GBI, we present a suitable duality for GBI along the lines of Esakia and Priestley and an algebraic proof of cut elimination in the setting of residuated frames of Galatos and Jipsen. We also show how the algebraic approach allows generic results on decidability, both positive and negative ones. In the final part of the paper, we gently introduce the substructural audience to some theory behind state-of-art tools, culminating with an algebraic and proof-theoretic presentation of (bi-) abduction.
\end{abstract}

\section{Contents}

1 Introduction

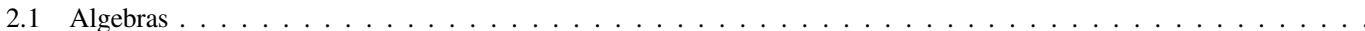

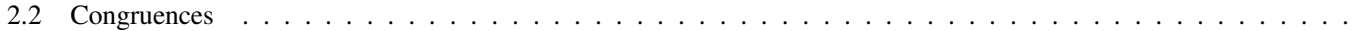

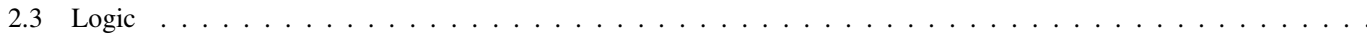

3 Concrete Models: Standard Models of BI $\ldots \ldots \ldots$

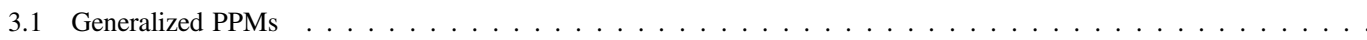

3.2 Intuitionistic vs. Classical Resource Models . . . . . . . . . . . . . . . . . . . . . . . . . .

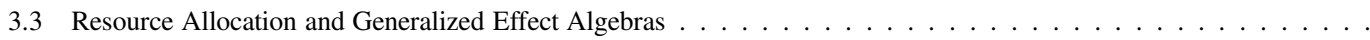

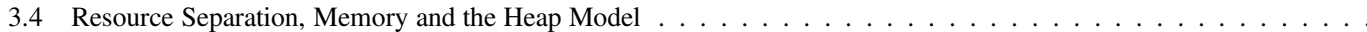

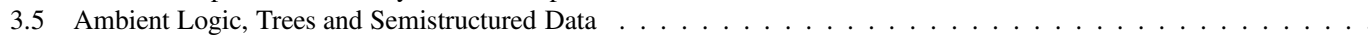

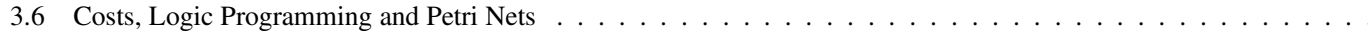

4 Essentially Noncommutative Models . . . . . . . . . . . . . . . . . . . . . . . . . . . . . . . .

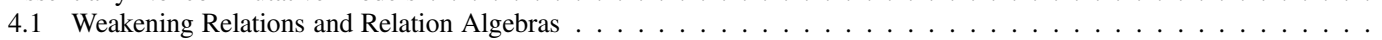

4.2 Language Models . . . . . . . . . . . . . . . . . . . . . . . . . . . . . . .

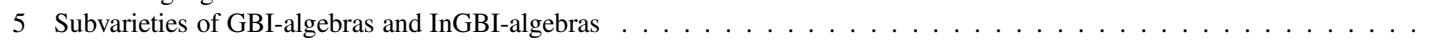

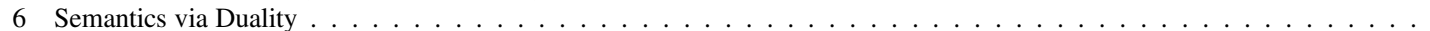

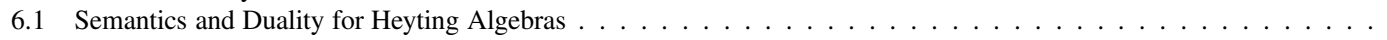

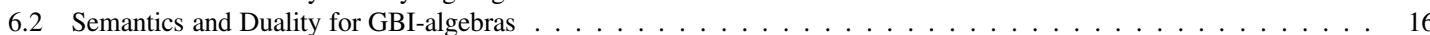

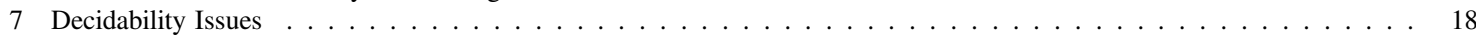

Peter Jipsen

School of Computational Sciences, Chapman University

E-mail: jipsen@chapman.edu

Tadeusz Litak

Informatik 8, FAU Erlangen-Nürnberg

E-mail: tadeusz.litak@fau.de 


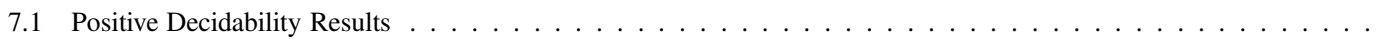

7.2 Subvarieties with Undecidable Equational Theory . . . . . . . . . . . . . . . . . . . .

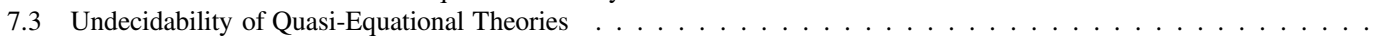

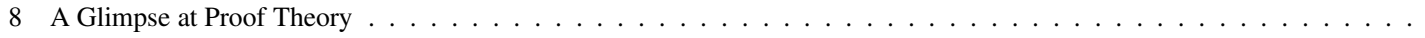

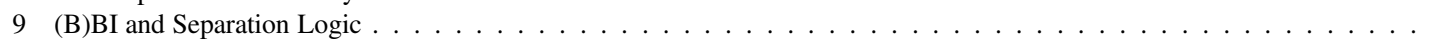

9.1 Basic Ideas of Floyd-Hoare Logic $(\mathrm{s}) \ldots \ldots \ldots \ldots \ldots$

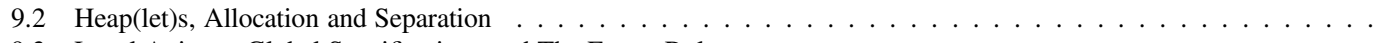

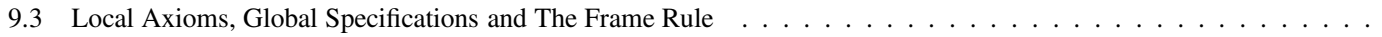

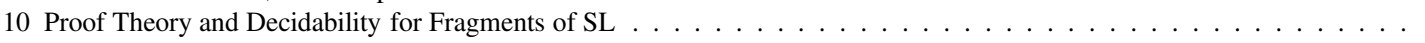

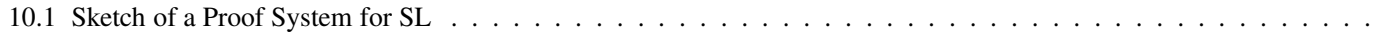

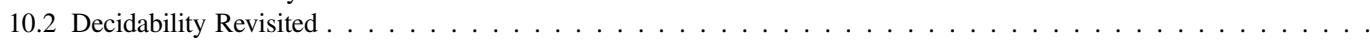

11 Bi-Abduction: The Main Issue of SL Proof Theory $\ldots \ldots \ldots \ldots$

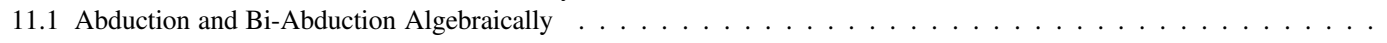

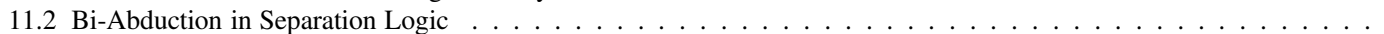

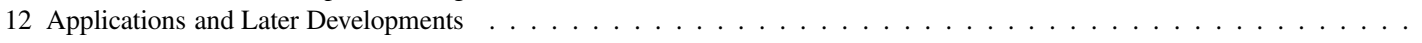

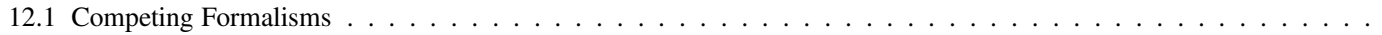

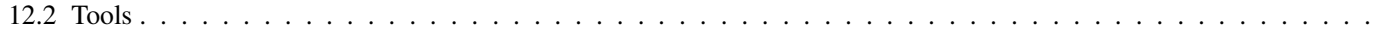

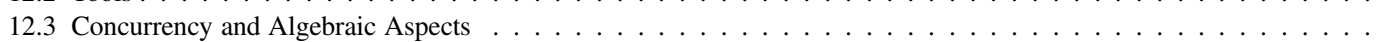

Given the size of this paper and possibly divergent interests of its readers, we tried to ensure a degree of independence between sections, even at the expense of certain redundancy.

\section{Introduction}

One of the major themes in the distinguished career of Hiroakira Ono, especially since the mid-1990s, has been the use of algebraic methods for substructural purposes. The monograph [GJKO07] which inspired the title of this overview is a monument to this approach. While for "standard" substructural logics we already have an impressive body of work, we believe the time has come to promote this approach for more applied systems, such as generalizations and extensions of the logic of Bunched Implications (BI).

The shortest description of BI is: take the (commutative) propositional substructural signature $\wedge, \vee, \top$, $\perp, *,-*, 1$ and add the Heyting implication $\rightarrow$ adjoint to the additive conjunction $\wedge$, immediately forcing the lattice structure to be distributive. The system was explicitly introduced by O'Hearn and Pym [OP99; OHe99; Pym99; Pym02]. In addition, Pym et al. [POY04], Brotherston et al. [BC10; BK14], O'Hearn [OHe12], Demri and Deters [DD15a] and this paper $(\S \S 34$ and $\S \S 9$ 12) present CS applications of BI and related formalisms such as separation logic. Perhaps the most important motivation can be summarized in one phrase: modular reasoning about the use of shared mutable data structures [Rey00; Rey02], i.e.,

structures where an updatable field can be referenced from more than one point. [Rey02]

In a narrow sense, this applies to heap mutation, pointer aliasing and (de)allocation: in short, dynamic memory management. Nevertheless, BI was originally proposed in the context of a broader investigation of "resource modelling" [POY04] (cf. [OPVH15]), with its "declarative" approach to resource contrasting with that of "proofs-as-actions" in linear logic. Unfortunately, we can only briefly discuss the most challenging application-i.e., shared-memory concurrency-in the concluding $\S 12.3$

Despite the rather recent history of BI, several of the key ideas have been around for a long time and some of them can be immediately recognized by substructural logicians. Moreover, the authors of pioneering BI papers made no secret of these substructural origins. Indeed, according to O'Hearn and Pym [Pym99, § 9], [OP99], [Pym02, Ch. 1] not only should BI be seen as a relevance logic, in fact up to minor syntactic details, an instance of Belnap's scheme of display logic [Bel82], but also the terminology bunches (for a structured term of formulas rather than a sequence of formulas) from which the very name of BI derives, comes from Dunn's work on a sequent calculus for the relevance logic R [Dun75; Bel82; Rea88]; cf. also the work of Mints [Min76].

On the mathematical side of things, another important early reference [Day70] shows that a monoidal (not necessarily closed) structure on a category induces a monoidal closed structure on the corresponding category of Set-presheaves. Together with Urquhart's work on the semantics of multiplicative intuitionistic linear logic [Ura72], this motivated total monoid semantics of O'Hearn and Pym [OP99; Pym99; Pym02], known to be incomplete in the presence of $\perp$ [Pym02, Prop. 4.8], [POY04, Prop. 6]. 
On the CS side of things, Reynolds [Rey00; Rey02] claims that the earliest motivation for separating conjunction is implicit in Burstall's [Bur72] early idea of a distinct nonrepeating tree system. To be more precise, rather than inspiring directly the earliest BI references, Burstall's work inspired Reynolds' development of a language of Hoare triples $(\S[$ ) for programs involving shared mutable data structures. Ishtiaq and O'Hearn [IO01] clarify the connection between that language, which came to be known as separation logic $(\S \S$ 9 12 ), and $\mathrm{BI}$ as the core of its assertional part; let us note here that this paper is very explicit about the substructural character of BI.

We hope to dispel here whatever remains of a prejudice which seemed to linger in the early days of BI, best illustrated by the following claim:

We are not looking for an algebraic semantics here, where one takes (say) a Heyting algebra with enough structure to model the multiplicatives; this would just be a collapsed version of the DCC semantics, and would not be very informative. [OP99, § 5]

It is worth noting that even a later influential paper by those authors [POY04 does find the need for an algebraic unifying framework when the focus is on theoremhood rather than, say, the structure of proofs.

More importantly, it is all too rarely mentioned that the core idea of BI-that of allowing not only multiplicative and additive conjunctions, but also their corresponding adjoint implications-has been seriously studied elsewhere, especially in the boolean setting. This was happening mostly in the 1990's for two convergent reasons. On the one hand, such research was motivated by generalizations of relation algebras, like in the $\mathrm{PhD}$ dissertation of the first author [Jip92]. On the other hand, it was inspired by a dynamic trend in formal semantics of natural language and in information processing. Volumes of collected papers from the period [MPM96] illustrate how fruitful this convergence was. The separation logic and BI communities seem largely unaware of this body of work and, at least in some cases, have proved overlapping results. Let us point out here just two examples, both of which can be traced back to the above-mentioned 1996 collection [MPM96] and which will be presented in more detail in $\S 7.2$.

- Algebraic results regarding (un)decidability for arrow logics [AKNSS96; KNSS95] overlap with results for boolean BI announced almost two decades later.

- Another example is provided by logic for layered graphs [CMP14], which essentially reinvents conjugated arrow logic [Mik96] (see Remark 7.5).

To unify these convergent lines of research, in $\S$ 2we take as our base variety the class GBI of generalized $\mathrm{BI}$ algebras, dropping the assumption of commutativity. In other words, we replace $*$ and $-*$ in the substructural reduct by $\cdot, \backslash$ and / (notation from the monograph [GJKO07]): clearly, one needs two residuals instead of one. This is not an uncommon step in the substructural setting; it is enough to recall how BL algebras were generalized to GBL algebras [JT02]. Here, we cover not only previously known commutative models of BI $(\S 3)$, but also weakening relations $(\S 4.1)$ or formal languages $(\S 4.2)$; moreover, we improve the treatment of labelled trees and semistructured data $(\S 3.5) .1$

$\S 5$ discusses systematically subvarieties of GBI, both those arising as generalizations of relation algebras and those obtained by adopting subvarieties of residuated lattices/FL-algebras [GJKO07, $\S 3.5]$ to our signature. In $\S 6$ we present a systematic approach to semantics via Esakia- and Priestley-style duality. $\S 7$ provides an overview of decidability and undecidability results for (quasi)equational theories. $\S[$ discusses an algebraic take on proof theory of $(\mathrm{G}) \mathrm{BI}$, in particular cut elimination; given the contributions of Hiroakira Ono to the literature on algebraic cut elimination [BJO04; GJKO07; GO10], this seems particularly natural material to present here. $\S 9$ is a gentle introduction to separation logic (SL) for an algebraically oriented audience. $\S 10$ reexamines the themes of $\S 7$ and $\S 8$ from the perspective of SL, sketching a suitable substructural proof system. In $\S 11$, we use the framework developed in earlier sections to suggest an algebraic and proof-theoretic approach to (bi-) abduction. Finally, $\S 12$ provides a short glimpse at applications and developments we could not cover in detail in this overview, especially state-of-the art tools or the treatment of concurrency.

\footnotetext{
${ }^{1}$ Let us note that dropping associativity of multiplicative conjunction has also been considered from all three perspectives, i.e., that of arrow logic, that of substructural logic, and most recently that of BI and resource reasoning. See Remark 7.5 for more information.
} 


\section{Logic and Algebra}

In this section, we discuss the basic algebraic setup and connection to Hilbert-style calculus. The main goal here is to present the class GBI of generalized (non-commutative) BI algebras as a general framework for our paper. However, even when it comes to standard, commutative BI-algebras, we believe the Hilbert-style axiomatization we provide in $\S 2.3$ has certain advantages over those presented in earlier BI literature.

\subsection{Algebras}

The algebras of bunched implication logic are Heyting algebras with a residuated commutative monoid. A Heyting algebra $\mathfrak{A}=(A, \wedge, \vee, \rightarrow, \top, \perp)$ is a bounded lattice $(A, \wedge, \vee, \top, \perp)$ s.t. $\rightarrow$ is the residual of $\wedge$, i.e.,

$$
x \wedge y \leq z \Longleftrightarrow y \leq x \rightarrow z \quad \text { for all } x, y, z \in A .
$$

It follows from this property that $\wedge$ distributes over $\vee$, hence $(A, \wedge, \vee)$ is a distributive lattice, which in turn implies that $\vee$ distributes over $\wedge$. In fact the residuation property easily implies the following stronger identities: if $\bigvee y_{i}$ and $\bigwedge y_{i}$ exist then

$$
x \wedge \bigvee y_{i}=\bigvee\left(x \wedge y_{i}\right), x \rightarrow \bigwedge y_{i}=\bigwedge\left(x \rightarrow y_{i}\right) \text { and }\left(\bigvee y_{i}\right) \rightarrow x=\bigwedge\left(y_{i} \rightarrow x\right)
$$

Heyting algebras are the algebraic semantics of intuitionistic logic, with (non-classical) negation defined by $\neg x=x \rightarrow \perp$.

A generalized bunched implication algebra (GBI-algebra), is a tuple $(A, \wedge, \vee, \rightarrow, \top, \perp, \cdot, \backslash, /, 1)$ where $(A, \wedge, \vee, \rightarrow, \top, \perp)$ is a Heyting algebra, $(A, \cdot, 1)$ is a monoid and $\backslash, /$ are the left and right residuals of $\cdot$, i.e.,

$$
x \cdot y \leq z \Longleftrightarrow y \leq x \backslash z \Longleftrightarrow x \leq z / y \quad \text { for all } x, y, z \in A
$$

We usually write $x y$ instead of $x \cdot y$, and assume that this operation has the highest priority, followed by $\backslash, /$, then $\wedge, \vee$, and finally $\rightarrow$. The residuation property implies that for all existing meets and joins

$$
\begin{aligned}
& x\left(\bigvee y_{i}\right)=\bigvee x y_{i} \quad x \backslash \bigwedge y_{i}=\bigwedge x \backslash y_{i} \quad\left(\bigvee y_{i}\right) \backslash x=\bigwedge y_{i} \backslash x \\
& \left(\bigvee y_{i}\right) x=\bigvee y_{i} x \quad\left(\bigwedge y_{i}\right) / x=\bigwedge y_{i} / x \quad x /\left(\bigvee y_{i}\right)=\bigwedge x / y_{i}
\end{aligned}
$$

GBI-algebras have distributive residuated lattices as reducts. Many other properties and results follow simply from this observation [GJKO07].

GBI-homomorphisms are functions that preserve all the operations, i.e., $f: A \rightarrow B$ such that $f(x \bullet$ $y)=f(x) \bullet f(y)$ for all $x, y \in A, \bullet \in\{\wedge, \vee, \rightarrow, \cdot, \backslash, /\}, f(\perp)=\perp$ and $f(1)=1(f(\top)=\top$ follows since $\perp \rightarrow \perp=\top)$.

The classes of Heyting algebras and of GBI-algebras are denoted by HA and GBI respectively. They are both finitely based equational classes, meaning they are determined by finitely many equations (or inequations, since $s \leq t$ and $s=s \wedge t$ are equivalent). For $\mathrm{HA}$ it suffices to take the equations of bounded lattices $(A, \wedge, \vee, \top, \perp)$ together with

$$
x \leq y \rightarrow((x \wedge y) \vee z) \quad x \wedge(x \rightarrow y) \leq y
$$

and for GBI-algebras one can add the inequations

$$
x \leq(x y \vee z) / y \quad((x / y) \wedge z) y \leq x \quad x \leq y \backslash(y x \vee z) \quad x((x \backslash y) \wedge z) \leq y .
$$

By Birkhoff's $\mathbb{H S P}$-theorem, equational classes are precisely the classes that are varieties, i.e., closed under homomorphic images $(\mathbb{H})$, subalgebras $(\mathbb{S})$ and direct products $(\mathbb{P})$, and moreover, for any class $K$ of algebras (of the same type) the variety $\mathbb{V}(K)$ generated by $K$ is $\mathbb{H} \mathbb{S P}(K)$ (for details cf., e.g., [Rafar]).

The variety of bunched implication algebras (or BI-algebras) is the subclass BI of all commutative GBIalgebras. In this case we use the more traditional notation of BI logic: $x * y=x y$ and $x-* y=x \backslash y=y / x$. The subvariety of Boolean BI-algebras, axiomatized by $\neg \neg x=x$, is denoted by BBI. 


\subsection{Congruences}

An equivalence relation $\theta$ on an algebra $\mathfrak{A}$ is a congruence if

$$
x \theta y \text { implies } f\left(z_{1}, \ldots, x, \ldots, z_{n}\right) \theta f\left(z_{1}, \ldots, y, \ldots z_{n}\right)
$$

for each argument of all fundamental operations $f$ of $\mathfrak{A}$. The set of all such congruences, ordered by inclusion, forms the congruence lattice $\operatorname{Con}(\mathfrak{A})$. The structure of this lattice determines several interesting properties of the algebra and of the class containing the algebras [Rafar] so we now consider how to determine congruences of GBI-algebras.

An algebra with a constant operation $e$ is called $e$-congruence regular if every congruence relation $\theta$ is determined by its $e$-congruence class $[e]_{\theta}=\{x: x \theta e\}$, i.e.,

$$
\text { for all } \theta, \psi,[e]_{\theta}=[e]_{\psi} \Longrightarrow \theta=\psi \text {. }
$$

In such an algebra it suffices to describe the $e$-congruence classes, and the poset of these classes, ordered by inclusion, is isomorphic to the congruence lattice of the algebra.

For example, groups are 1-congruence regular and Heyting algebras are $T$-congruence regular, whereas monoids and lattices (even with bounds and distributivity) are not $e$-congruence regular with respect to any constant operation $e$. In the case of Heyting algebras, the $T$-congruence classes are precisely the latticefilters of the algebra, i.e., sets $F \subseteq A$ such that $\uparrow F \subseteq F$ and $x, y \in F$ imply $x \wedge y \in F$. The congruence $\theta_{F}$ associated with the filter $F$ is defined by $x \theta_{F} y \Longleftrightarrow x \rightarrow y, y \rightarrow x \in F$.

If we ignore the Heyting operations $\rightarrow, \top, \perp$ then GBI-algebras are residuated lattices. Congruence classes of lattices are always convex (i.e., if $x \leq y \leq z$ and $x, z$ are in a class, then $y$ is also in the class), and as mentioned above, residuated lattices are 1-congruence regular (see e.g. [JT02]). The 1-congruence classes are precisely the convex subalgebras $\mathfrak{C}$ that are closed under conjugation, i.e., for all $a \in A$ and all $x \in C$ it follows that $a \backslash x a \wedge 1, a x / a \wedge 1 \in C$. Given such a congruence class $C$, the congruence $\theta_{C}$ is determined by $x \theta_{C} y \Longleftrightarrow x \backslash y \wedge y \backslash x \wedge 1 \in C$.

From these observations we conclude that GBI-algebras are both 1-congruence regular and $\top$-congruence regular. The following criterion can be used to check whether a residuated lattice congruence is a GBIcongruence.

Theorem 2.1 Suppose $\theta$ is a residuated lattice congruence on the $\rightarrow, \perp, \top$-free reduct of a GBI-algebra $\mathfrak{A}$. Then the following are equivalent:

1. $\theta$ is a GBI-congruence.

2. For all $x, y, z \in A$ if $x \theta y$ then $x \rightarrow z \theta y \rightarrow z$ and $z \rightarrow x \theta z \rightarrow y$.

\subsection{Logic}

Throughout we use algebraic term syntax for logical formulas. Propositional intuitionistic logic uses the symbols $\wedge, \vee, \rightarrow, \top, \perp$ to build formulas (= terms) from variables $x, y, z, x_{1}, \ldots$, and $x \leftrightarrow y$ abbreviates the formula $(x \rightarrow y) \wedge(y \rightarrow x)$. The consequence relation $\vdash_{\mathrm{IL}}$ of intuitionistic logic is defined by the following Hilbert system, traditionally denoted by HJ. The axioms are all substitution instances of the formulas below, and modus ponens is the only inference rule:

$$
\begin{gathered}
\frac{x \quad x \rightarrow y}{y} \quad \perp \rightarrow x \quad x \rightarrow \top \\
x \rightarrow(y \rightarrow x) \quad(x \rightarrow(y \rightarrow z)) \rightarrow((x \rightarrow y) \rightarrow(x \rightarrow z)) \\
x \wedge y \rightarrow x \quad x \wedge y \rightarrow y \quad x \rightarrow(y \rightarrow x \wedge y) \\
x \rightarrow x \vee y \quad x \rightarrow y \vee x \quad(x \rightarrow z) \rightarrow((y \rightarrow z) \rightarrow(x \vee y \rightarrow z))
\end{gathered}
$$

Given a set $\Gamma$ of formulas, $\Gamma \vdash_{\mathrm{IL}} \varphi$ holds if there is a finite sequence of formulas $\varphi_{1}, \ldots, \varphi_{n}=\varphi$ such that each $\varphi_{i}$ is in $\Gamma$, is an axiom, or is the result of modus ponens applied to $\varphi_{j}, \varphi_{k}$ for some $j, k<i$. For example, 
a standard (but rather non-obvious) deduction shows that $\rightarrow$ is transitive: $\{x \rightarrow y, y \rightarrow z\} \vdash_{\mathrm{IL}} x \rightarrow z$. The theorems (tautologies) of IL are all the formulas $\varphi$ such that $\emptyset \vdash_{\mathrm{IL}} \varphi$, or equivalently $\vdash_{\mathrm{IL}} \varphi \leftrightarrow T$.

$\mathrm{HJ}$ is extended to a Hilbert system HGBI for GBI by adding symbols $\cdot, \backslash, /$, all substitution instances of the following formulas as axioms

$$
(x y) z \leftrightarrow x(y z) \quad 1 x \leftrightarrow x \quad x 1 \leftrightarrow x
$$

and the bidirectional residuation rules

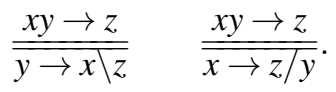

While many other axioms (or rules) can be used to axiomatize $\mathrm{GBI}$, the axiomatization given here emphasizes the close relationship between Hilbert systems (of sufficient strength) and equational deduction. Neither approach is as effective as the sequent calculus decision procedure that is outlined in $\S 8$, but Birkhoff's system of equational deduction requires perhaps less explanation than the corresponding logical systems, and it allows substitution of equal terms based on equalities derived from the axioms or assumptions.

Theorem 2.2 HGBI corresponds to GBI: $\Gamma \vdash_{H B G I} \varphi$ if and only if $\varphi=\top$ can be derived by equational reasoning from $\{\gamma \leftrightarrow \top: \gamma \in \Gamma\}$.

Proof Analogous results are well known for intuitionistic and substructural reducts of GBI; cf. Remark 2.3 The HGBI axioms clearly correspond to the equational monoid axioms. It remains to show that Birkhoff's congruence rules are derivable in HGBI. For example the rule $\frac{x \rightarrow y}{y \backslash z \rightarrow x \backslash z}$ is proved as follows:

$$
\begin{aligned}
& \begin{array}{llll}
\text { 1. } x \rightarrow y & \text { 2. } y \backslash z \rightarrow y \backslash z \quad \text { 3. } y(y \backslash z) \rightarrow z \quad \text { 4. } y \rightarrow z /(y \backslash z)
\end{array} \\
& \text { 5. } x \rightarrow z /(y \backslash z) \quad \text { 6. } x(y \backslash z) \rightarrow z \quad \text { 7. } y \backslash z \rightarrow x \backslash z
\end{aligned}
$$

where transitivity of $\rightarrow$ was used for step 5. The rules $\frac{x \leftrightarrow y}{z \backslash x \leftrightarrow z \backslash y}, \frac{x \leftrightarrow y}{x z \leftrightarrow y z}, \frac{x \leftrightarrow y}{z x \leftrightarrow z y}, \frac{x \leftrightarrow y}{x / z \leftrightarrow y / z}, \frac{x \leftrightarrow y}{z / y \leftrightarrow z / x}$ are proved similarly.

A Hilbert system HBI for bunched implication logic is obtained by adding an axiom $x * y \rightarrow y * x$, in which case the rules for / can be omitted, and the rules for $\backslash$ are rewritten with $-*$. The resulting system is similar to the one in [Pym02]. Alternatively one can use the following system with two more axioms and simpler rules (in addition to the axioms and rules of $\mathrm{HJ}$ ).

$$
\begin{gathered}
(x * y) * z \leftrightarrow x *(y * z) \quad x * y \rightarrow y * x \quad x * 1 \leftrightarrow x \quad x *(x-* y) \rightarrow y \\
x-*(y-* z) \leftrightarrow x * y-* z \quad \frac{x \rightarrow y}{x * z \rightarrow y * z} \quad \frac{x \rightarrow y}{1 \rightarrow x-* y} .
\end{gathered}
$$

Remark 2.3 The connection between HGBI (HBI) and GBI (BI) exposed by Theorem 2.2 is an instance of the phenomenon known as algebraizability [BP89]. In this volume, an overview is provided by Font [Fonar]. For Heyting and substructural reducts of (G)BI, details can be found, e.g., in Galatos et al. [GJKO07, $\S \S 1.4 .3,2.6]$. In fact, due to the presence of Heyting $\rightarrow$, logics extending HGBI belong to a particularly well-behaved class of Rasiowa implicative logics [CN10; Ras74] [Fonar, $§$ 5].

\section{Concrete Models: Standard Models of BI}

As we already suggested in $\S 1$ GBI admits a wealth of practically motivated models. In this section, we focus on models previously investigated in the commutative setting, using Pym et al. [POY04, $\S 4$ ] as our blueprint. Even here, we are going to see potential for non-commutative generalizations (cf. especially $\S$ 3.5]; we are going to explore more "natively non-commutative" models in $\S$ 4 


\subsection{Generalized PPMs}

In order to streamline the discussion and facilitate checking the GBI axioms for a large class of examples, let us follow the example of Pym et al. [POY04, § 3.6] (see also [GMP05]) and define a convenient semantics

- whose defining properties are easily verifiable,

- which covers many natural models and yet

- avoids the full generality of semantics for distributive substructural logics based on ternary relations.

More specifically, consider preordered partial monoids $\mathfrak{M}=(M, \cdot E, \sqsubseteq)$ with $\cdot$ lifted to an operation on subsets by $X \cdot Y=\{z \mid \exists x \in X, y \in Y . x \cdot y \sqsubseteq z\}$ for $X, Y \subseteq M$ and with

- $(M, \cdot)$ being a partial semigroup up to the equivalence relation $\equiv$ defined as $\sqsubseteq \cap \sqsupseteq$, i.e., whenever one element of $\{x \cdot(y \cdot z),(x \cdot y) \cdot z\}$ exists, the other one exists as well, and they are in the same $\equiv$-class,

- $E \subseteq M$ being a collection of unit elements, i.e., for any $x \in M$,

$$
\emptyset \neq\{x\} \cdot E \subseteq[x]_{\equiv} \quad \emptyset \neq E \cdot\{x\} \subseteq[x]_{\equiv}
$$

(we always assume the closure of $E$ under $\equiv$ ),

- the bifunctoriality condition

$$
x \sqsubseteq x^{\prime}, y \sqsubseteq y^{\prime} \text { and } x^{\prime} \cdot y^{\prime} \in M \text { implies } x \cdot y \in M \text { and } x \cdot y \sqsubseteq x^{\prime} \cdot y^{\prime}
$$

holding for any $x, x^{\prime}, y, y^{\prime} \in M$.

Such a structure will be called a generalized PPM (short for preordered partial monoid), and the notation $x \cdot y \in M$ is used to indicate that $x \cdot y$ is defined. Most of the time, we will restrict attention to the case where $E=\{\mathrm{e}\}$ for some e $\in M$; in such a case, we will speak of a (proper) PPM. Define now $\mathfrak{M}^{+}$, the complex algebra of $\mathfrak{M}$, as the algebra in GBI-signature whose

- universe consists of all upsets of $(M, \sqsubseteq)$,

- Heyting connectives are interpreted in the standard intuitionistic way,

- the unit element is defined as the upset of $E$,

$-X \cdot Y$ is as defined above,

- the residuals are obtained using the fact that upsets are closed under arbitrary unions and - distributes over these unions.

Fact 3.1 The complex algebra of any PPM is a GBI-algebra.

This is a straightforward generalization of the facts used by Pym et al. [POY04] and Galmiche et al. [GMP05] with the obvious difference that we are not assuming commutativity. Of course, from the point of view of a substructural logician, such partial monoids can be turned into instances of ternary relation semantics by setting $R x y z$ whenever $x \cdot y=z$. We will return to relational semantics in $\S 6.2$ and $\S 8$, each time with a somewhat different focus and somewhat different notation.

\subsection{Intuitionistic vs. Classical Resource Models}

Most PPM-style models discussed below can be in fact obtained in two flavours: a monoid with a degenerate or discrete order (thus yielding a boolean GBI-algebra) and an associated intuitionistic structure with a nontrivial order definable in terms of the monoid operation. This has been noted early on in the development of BI logic, leading to Gödel-McKinsey-Tarski-style modal translations between intuitionistic and classical logics of suitable classes of models [IO01, Prop. 9] (see also [GL06]) and commonly used terms intuitionistic semantics and intuitionistic assertion

\footnotetext{
${ }^{2}$ Interestingly, the intuitionistic BI is embeddable in the classical BBI [LG09], rather than the other way around. Indeed, as pointed out in Litak et al. [LPR17, $\S 4.1$ ], (un)decidability results discussed in $\S 7$ entail that no negative translation from BBI to BI can work.
} 
Thus, the idea is well known, but in our setting we can present it in a rather convenient way. Let a PME (a partial monoid up to equivalence) be a generalized PPM where the ordering is an equivalence relation. Obviously, this happens iff $\sqsubseteq$ coincides with $\equiv$ as defined above.

We speak of CPME (commutative partial monoid up to equivalence) when the commutativity law $x \cdot y \equiv y \cdot x$ holds. While commutativity makes transition from the boolean to the intuitionistic setting much smoother, we can do without it, at the expense of introducing some additional apparatus. Given a (not necessarily commutative!) PME $\mathfrak{M}=(M, \cdot, E, \equiv)$, let $C_{\mathfrak{M}}$ be the collection of those $x \in M$ for which

- for any $y \in M$, whenever $x \cdot y \in M$, there exists $y^{\prime}$ s.t. $x \cdot y \equiv y^{\prime} \cdot x$ and

- for any $y \in M$, whenever $y \cdot x \in M$, there exists $y^{\prime}$ s.t. $y \cdot x \equiv x \cdot y^{\prime}$.

Fact 3.2 In any $P M E \mathfrak{M}=(M, \cdot, E, \equiv)$ :

(i) $E \subseteq C_{\mathfrak{M}}$,

(ii) $C_{\mathfrak{M}} \cdot C_{\mathfrak{M}} \subseteq C_{\mathfrak{M}}$, that is, $C_{\mathfrak{M}} \subseteq C_{\mathfrak{M}} \backslash C_{\mathfrak{M}}$,

(iii) For any $X \in \mathfrak{M}^{+}, X \cdot C_{\mathfrak{M}}=C_{\mathfrak{M}} \cdot X$ and $C_{\mathfrak{M}} \backslash X=X / C_{\mathfrak{M}}$,

(iv) Whenever $\mathfrak{M}$ is commutative, $C_{\mathfrak{M}}=M$.

Now let us define the substate relation 3 , generalizing the corresponding definition for separation algebras (see below) proposed by Calcagno et al. [COY07]:

$$
x \preceq_{\mathrm{s}} y \quad \text { iff } \quad \exists z \in C_{\mathfrak{M}} \cdot x \cdot z \equiv y .
$$

Theorem 3.3 Let $\mathfrak{M}=(M, \cdot, E, \equiv)$ be a PME. Then

(i) the complex algebra $\mathfrak{M}^{+}$of unions of equivalence classes is a boolean GBI-algebra,

(ii) $\preceq_{\mathrm{s}}$ is a preorder, with the associated equivalence relation $\equiv_{\mathrm{s}}=\preceq_{\mathrm{s}} \cap \succeq_{\mathrm{s}}$ containing the original $\equiv$,

(iii) $\mathfrak{M}_{\mathrm{s}}=\left(M, \cdot, E, \preceq_{\mathrm{s}}\right)$ is a generalized PPM and hence $\left(\mathfrak{M}_{\mathrm{s}}\right)^{+}$is a GBI-algebra,

(iv) Elements of $\left(\mathfrak{M}_{\mathrm{s}}\right)^{+}$are exactly those sets A of equivalence classes in $\mathfrak{M}$ which in $\mathfrak{M}^{+}$satisfy one of the following equivalent conditions:

$A \subseteq A / C_{\mathfrak{M}}, \quad C_{\mathfrak{M}} \subseteq A \backslash A, \quad A \cdot C_{\mathfrak{M}} \subseteq A, \quad A \cdot C_{\mathfrak{M}}=A$,

$A \subseteq C_{\mathfrak{M}} \backslash A, \quad C_{\mathfrak{M}} \subseteq A / A, \quad C_{\mathfrak{M}} \cdot A \subseteq A, \quad C_{\mathfrak{M}} \cdot A=A$.

(v) For any $A, B \in\left(\mathfrak{M}_{\mathrm{s}}\right)^{+}$s.t. $B \subseteq C_{\mathfrak{M}} / C_{\mathfrak{M}}$ (where $C_{\mathfrak{M}} / C_{\mathfrak{M}}$ denotes an element of $\mathfrak{M}^{+}$rather than $\left.\left(\mathfrak{M}_{\mathrm{s}}\right)^{+}\right), A \cdot B \leq A$ holds in $\left(\mathfrak{M}_{\mathrm{s}}\right)^{+}$. Hence, whenever $\mathfrak{M}$ is a CPME, $\left(\mathfrak{M}_{\mathrm{s}}\right)^{+} \in \mathrm{BI}_{\mathrm{w}}(c f . \S 5)$.

\section{Proof}

(i) A direct corollary of Fact 3.1 unions of equivalence classes are upsets of PME and they are closed under complementation.

(ii) Transitivity of $\preceq_{s}$ follows from associativity of · and Fact 3.2 (ii), whereas reflexivity of $\preceq_{s}$ follows from the monoidal unit law and Fact 3.2.(i) (both up to bifunctoriality of $\equiv$ and equivalence). The latter assures also the containment claim, jointly with transitivity of $\equiv$.

(iii) In the light of (ii), we only need to ensure bifunctoriality of $\preceq_{s}$; note that this is the first time when we use the fact that the substate relation is defined in terms of $C_{\mathfrak{M}}$. Assume $z_{x}, z_{y} \in C_{\mathfrak{M}}$ s.t. $x \cdot z_{x} \equiv x^{\prime}$ and $y \cdot z_{y} \equiv y^{\prime}$. By bifunctoriality of $\equiv$, we get that $\left(x \cdot z_{x}\right) \cdot\left(y \cdot z_{y}\right) \equiv x^{\prime} \cdot y^{\prime}$. Iterating the associativity law yields $x \cdot\left(z_{x} \cdot y\right) \cdot z_{y} \equiv x^{\prime} \cdot y^{\prime}$. Now we use the definition of $C_{\mathfrak{M}}$ to pick a suitable $z_{x}^{\prime}$ s.t. $x \cdot y \cdot z_{x}^{\prime} \cdot z_{y} \equiv x^{\prime} \cdot y^{\prime}$. Thanks to Fact 3.2.(ii), we obtain that $z_{x}^{\prime} \cdot z_{y} \in C_{\mathfrak{M}}$.

(iv) By definition, a set of equivalence classes $A$ is an element of $\left(\mathfrak{M}_{\mathrm{s}}\right)^{+}$iff for any $a \in A$ and $c \in C_{\mathfrak{M}}$, it is the case that $a \cdot c \in A$. This is an equivalent way of stating that $\mathfrak{M}^{+}$, it holds that $A \cdot C_{\mathfrak{M}} \subseteq A$. The rest follows form Fact 3.2.(iii).

(v) Assume $A, B \in\left(\mathfrak{M}_{\mathrm{s}}\right)^{+}, B \subseteq C_{\mathfrak{M}}$ and $x \in A \cdot B$. That is, there are $a \in A, b \in B, z \in C_{\mathfrak{M}}$ s.t. $a \cdot b \cdot z \equiv x$. By assumption, we get that $b \cdot z \in C_{\mathfrak{M}}$, thus $a \preceq_{\mathrm{s}} x$ and we just use the fact that $A$, like all elements of $\left(\mathfrak{M}_{\mathrm{s}}\right)^{+}$, is $\preceq_{\mathrm{s}}$-upward closed.

\footnotetext{
${ }^{3}$ In the theory of semigroups, one would rather use the name algebraic preordering. It is also known as divisibility relation for commutative semigroups.
} 
One problem with this construction is that $\equiv_{\mathrm{s}}$ may happen to be bigger than the original $\equiv$, also when $\equiv$ is just the diagonal (equality relation). Let us say that a PME

- is right-cancellative if $x \cdot y \equiv x \cdot y^{\prime}$ implies $y \equiv y^{\prime}$ (in the presence of commutativity this implies leftcancellativity) and

- satisfies indivisibility of units if $x \cdot y \in E$ implies $x \in E$ (and hence also $y \in E$ ).

Theorem 3.4 Let $\mathfrak{M}=(M, \cdot, E, \equiv)$ be a PME. Whenever $\mathfrak{M}$ is right-cancellative and satisfies indivisibility of units, then the associated equivalence relation $\equiv_{\mathrm{s}}=\preceq_{\mathrm{s}} \cap \succeq_{\mathrm{s}}$ of $\mathfrak{M}_{\mathrm{s}}$ is the same as the original $\equiv$.

Proof We have already established in Theorem 3.3 (ii) that $\equiv_{\mathrm{s}}$ contains the original $\equiv$. Thus, we have only to show the converse inclusion. Assume then that $z_{x}, z_{y} \in C_{\mathfrak{M}}, x \cdot z_{x} \equiv y$ and $y \cdot z_{y} \equiv x$. Therefore, $x \cdot z_{x} \cdot z_{y} \equiv x \cdot e$. Right-cancellativity implies that $z_{x} \cdot z_{y} \equiv e$ and indivisibility of units implies that $z_{x} \in E$, hence $x \equiv y$.

Whenever $\equiv$ in $\mathfrak{M}$ is the equality relation (as it happens in most natural examples), Theorem 3.4 says that cancellativity and indivisibility of units of $\mathfrak{M}$ entail that $\preceq_{\mathrm{s}}$ is a partial ordering.

Separation algebras [COY07] are CPME's (i.e., commutative PME's) which moreover are cancellative, have $E=\{e\}$, i.e., are (proper) PPMs in our terminology, and where $\equiv$ is just the identity relation.

We are now ready to instantiate this framework to specific applications.

\subsection{Resource Allocation and Generalized Effect Algebras}

Given any set (which is thought of as the supply of resources), one can impose a separation algebra structure on the set of all its subsets by taking $x \cdot y$ to be $x \cup y$ whenever these two sets are disjoint and undefined otherwise. The order can be taken to be discrete (equality) or one can transfer it via Theorems 3.3 and 3.4 obtaining ordinary inclusion relation as the ordering. The empty set is the identity element, and the collection of finite subsets forms a subalgebra. This example is discussed in detail in $\S 4.3$ of Pym et al. [POY04] (see also [IO01; Rey00; Rey02]).

A generalized effect algebra is a separation algebra that satisfies the positivity law: if $x \cdot y=e$ then $x=$ $e=y$. This holds, e.g., for the separation algebra defined by disjoint union. The more specialized concept of effect algebra was defined by Foulis and Bennett [FB94] as an abstraction of quantum effect operators in Hilbert space (i.e., self-adjoint operators with spectrum in the unit interval). These are generalized effect algebras with a constant $\top$ satisfying in addition the orthosupplementation law: for every $x$, there exists a unique $y$ s.t. $x \cdot y$ exists and $x \cdot y=\top$.

\subsection{Resource Separation, Memory and the Heap Model}

The next example follows the same idea as the separation algebra given by disjoint union [IO01; Rey00; Rey02; POY04; OHe12; DD15a]. It is also probably the one most responsible for the success of BI in computer science. This time, resources are interpreted concretely as portions of computer memory. A good overview of various possible notions of memory models can be found in the recent work of Brotherston and Kanovich [BK14, § 2] and also in Demri and Deters [DD15a].

More specifically, given an infinite set of locations $L$ and a set of record values $R V$, the latter possibly with some additional structure, we define heaps (or heaplets, as suggestively named by Berdine et al. [BCO06]) as finite partial functions from $L$ to $R V$. Particularly when reasoning about linked data structure, it is common to demand that we have in addition a function from $R V$ to $L \cup\{$ nil $\}$, where nil is a fixed null pointer. Actually, separation logic overviews quite often restrict attention to single-linked lists, defining $R V$ to be $V \times(L \cup\{$ nil $\})$ and the set of base values $V$ is typically taken to be, e.g., $\mathbb{Z} \cup L \cup\{$ nil $\})$. There are other possible choices for $R V$, for example it can be taken to be $V^{2}$.

One obtains a separation algebra structure on heaps by setting $h \cdot h^{\prime}$ to be their union when domains of $h$ and $h^{\prime}$ are disjoint and undefined otherwise. Again, the intuitionistic option offered by Theorems 3.3 and 3.4 orders heaps by inclusion between their graphs. Interestingly, one of the earliest papers on separation logic [Rey00] took the latter route (cf. also [IO01; Rey02; POY04]). 
Let us mention here one more possible tweak to these models, which makes them closer to memory models of actual programming languages and more convenient from the point of view of development of program logics as discussed in $\S 9$. It is also our first opportunity to use generalized rather than proper PPM's. Namely, assume that in addition to the collection of locations $L$, we also have a collection of ordinary program variables PVar, and in addition to record values $R V$, we also have store values (or stack values) Val. We define then stores (or stacks) as mappings from PVar to Val, either total or (finite) partial ones 4 and the store-and-heap (or stack-and-heap) model [BK14, § 2.2]) as consisting of pairs $(s, h)$ with $s$ a store and $h$ a heap. The set of units $E$ is defined then as the collection of all pairs $(s, \emptyset)$. We say $(s, h) \cdot\left(s^{\prime}, h^{\prime}\right)$ is defined whenever $s=s^{\prime}$ and $h \cdot h^{\prime}$ (as introduced above) is defined. As we are going to see in $\S 9$ having stores at our disposal we do not need anymore the above-mentioned restrictions on the structure of $R V$ such as the one that each $R V$ should contain a pointer to another $R V$.

\subsection{Ambient Logic, Trees and Semistructured Data}

Pym et al. [POY04, § 4.2] illustrate how to obtain a PPM using Cardelli and Gordon's ambient logic [CG00]. This influential formalism was developed further in a number of references, some of them focusing on reasoning about trees and semistructured data [CG04]. Subsequent developments included context logic, a formalism specifically intended for analyzing dynamic updates to tree-like structures with pointers (such as XML with identifiers and idrefs) [CGZ05; CDG10]. As this example generalizes particularly nicely to the non-commutative setting, we discuss it in more detail.

Consider a set of labels Lab. The set of labelled trees (which might be more adequately called labelled forests in the terminology of W3C specifications [CG04, §3.4]) is given by the following syntax:

$$
S, T::=0|a[S]| S \cdot T
$$

where $a \in L a b$. This is a standard way to represent semistructured data like XML documents. One identifies forests using the equivalence relation generated by associativity of · and 0 being a neutral element for ·, which obviously yields a generalized PPM. In this free construction of labelled trees, the operation · is total and indeed it was intended to be total in several references [CG04; POY04], but as pointed out by e.g. Calcagno et al. [CGZ05, $\S 2$ ], it is natural to restrict the attention to trees with uniquely identifying labels. Under such an assumption, $S \cdot T$ is defined only if the labels occurring in $S \cdot T$ are disjoint-and thus we have yet another example of a partially defined monoid.

However, from our point of view it is even more interesting to note that while almost all references mentioned in this subsection insists on commutativity of ·, it is hardly the most obvious assumption. In fact, not only are XML documents defined as finite sibling-ordered trees, but official specifications of languages standardized by W3C for the purpose of querying and navigating XML documents like XPath and XQuery allows explicit access to the sibling order (see, e.g., ten Cate et al. [CLM10; CFL10] for more information, including a discussion of the relationship of these formalisms to modal logics). And, needless to say, any representation of trees for storage or manipulation purposes would involve ordering on nodes; in short, · should be thought of as creating lists rather than multisets. While this issue is occasionally discussed in the literature [CG04, $\S \S 3.1 \& 3.4$ ], most references tend to glide over this problem. Dropping the requirement of commutativity makes the complex algebra of such a PPM an instance of a GBI algebra which is not a BI algebra.

Finally, let us note that one obtains a GBI algebra with a non-boolean Heyting reduct by replacing the discrete order on trees by $S \sqsubseteq T$ defined as " $S$ is a generated subtree (or, strictly speaking, a generated subforest) of $T$ ".

\footnotetext{
${ }^{4}$ Brotherston and Kanovich [BK14, § 2.2] stick to the finite partial definition, but the total one is arguably more natural and common (see e.g. [Pie+18; Win93; DD15a]); this is one of differences between stores (stacks) and heap(let)s. Especially under the total perspective the name store, used also by Demri and Deters [DD15a] seems more adequate. Nevertheless, both perspectives can be brought together: one can think of the constant function $\lambda x:$ PVar.0 as the default or unintialized stack (store) and restrict the attention to stacks almost everywhere equal to zero.
} 


\subsection{Costs, Logic Programming and Petri Nets}

Pym et al. [POY04, $§ 4]$ describe three other classes of CS-motivated PPM's giving rise to natural BI complex algebras. In brief, they are as follows:

- an adjustment of the Petri net semantics of linear logic described by Engberg and Winskel [EW97]. An interesting feature of this example is that the PPM in question illustrates the benefits of allowing preorders instead of insisting on posets. Modelling of Petri nets using separation algebras is discussed by Calcagno et al. [COY07, $\S 2]$;

- a logic programming model of Armelín and Pym [AP01] based on a commutative total PPM of hereditary Harrop bunches and

- a money and cost example, tailored to highlight both similarities and differences with Girard's Marlboros and Camels linear logic example.

Brotherston and Calcagno [ $\mathrm{BC} 10, \S 5]$ provide some additional commutative models, focusing on involutive boolean ones, i.e., those whose dual algebras belong to the variety denoted in $\S[5$ as InBBI (Brotherston and Calcagno [BC10] use the term classical).

\section{Essentially Noncommutative Models}

Finally, we present two more classes of examples, illustrating the advantages of dropping the assumption of commutativity even more starkly than $\S 3.5$

\subsection{Weakening Relations and Relation Algebras}

This example involves generalized PPM's. Consider a poset $\mathfrak{X}=(X, \sqsubseteq)$. Say that $R$ is a weakening relation on $\mathfrak{X}$ iff $\sqsubseteq \circ R \circ \sqsubseteq=R$, where $\circ$ is the relation composition. The collection of all the weakening relations on $\mathfrak{X}$ is written as $w$ Rel $\mathfrak{X}$.

Fact 4.1 wRelX is closed under arbitrary unions $\bigcup$ and intersections $\bigcap$, with $\circ$ distributing over $\bigcup$ and $\sqsubseteq$ being the neutral element of $\circ$.

Consequently, wRel $\mathfrak{X}$ carries the structure of a GBI algebra and is called the full weakening relation algebra generated by $\mathfrak{X}$.

It is possible to see $w$ Rel $\mathfrak{X}$ as a complex algebra of a (generalized!) PPM. Set $\mathfrak{X}^{\partial}=(X, \sqsupseteq)$ and consider $M=\mathfrak{X} \times \mathfrak{X}^{\partial}$. The set of unit elements $E$ and the partial monoid operation $\cdot$ are defined then in an obvious way, i.e., $E=\{(x, x) \mid x \in X\}$ and $(x, y) \cdot\left(y^{\prime}, z\right)=(x, z)$ whenever $y=y^{\prime}$ and undefined otherwise. Fact 4.1 is then obtained as a corollary of Fact 3.1

What is particularly interesting about this example is that when we restrict attention to discrete $\sqsubseteq$, we obtain exactly what is known as full set relation algebras [HMT85, Def. 5.3.2] or square relation algebras [Mad06, Ch. 6.0.3].

\subsection{Language Models}

Consider an alphabet $\Sigma$; as usual, we write the set of words in $\Sigma$ as $\Sigma^{*}$. The notions of language and regular language are standard and so is the notion of composition of languages. It is well known that the set of regular languages, just like of all languages, is closed under finite unions and intersections, residuals and boolean complementation (cf., e.g., [Pra91, § 3.2]). Therefore, both arbitrary languages and regular languages over a given $\Sigma$ form a nice example of a boolean GBI. In fact, we can see this as another instance of the PPM setting, but once again dropping the assumption of commutativity was crucial to achieve full generality. 


\section{Subvarieties of GBI-algebras and InGBI-algebras}

A subvariety of GBI (the variety of all GBI-algebras) is any subclass that is closed under $\mathbb{H} S \mathbb{S P}$, or equivalently any subclass that is determined by a set of identities (including the equational axioms of GBI). The collection of all subvarieties of GBI is denoted by $\Lambda_{\mathrm{GBI}}$ or simply $\Lambda$. Since subvarieties are determined by sets of identities, $\Lambda$ contains at most continuum many subvarieties. Jankov [Jan68] showed that this upper bound is reached by subvarieties of Heyting algebras, hence the same is true for GBI. Subvarieties are ordered by inclusion, and $\Lambda$ is in fact an algebraic distributive lattice, with $U \wedge W=U \cap W$ and $U \vee W=\mathbb{V}(U \cup W)$. The least element is the trivial variety $\mathrm{O}$ of one-element GBI-algebras, and the largest element is $\mathrm{GBI}$.

For an involutive GBI-algebra, we first need to expand the language with a new constant symbol 0 , which is used to term-define the linear negations $\sim x=x \backslash 0$ and $-x=0 / x$. Then we add the identities $\sim-x=x=-\sim x$ to define the variety $\ln \mathrm{GBI}$.

Some prominent subvarietie 5 of $\mathrm{GBI}$ and $\ln \mathrm{GBI}$ are:

- The variety BI defined relative to GBI by $x y=y x$.

- The variety $\mathrm{GBI}_{\mathrm{w}}$ of GBI-algebras that satisfy the structural rule of weakening, defined by the identity $x \cdot y \leq x$, or equivalently by $T=1$.

- The variety BGBI of Boolean GBI-algebras, defined by $\neg \neg x=x$. It is also known as RM, the variety of residuated Boolean monoids [Jip92].

- The variety CyGBI of cyclic involutive GBI algebras, defined relative to $\ln G B I$ by $\sim x=-x$.

- The variety $\mathrm{InBI}$ of involutive BI-algebras, defined relative to $\ln \mathrm{GBI}$ by $x y=y x$.

- The variety wRRA of weakening representable relation algebras, generated by all full weakening relation algebras (cf. $\S$ 4.1).

- The variety SeA of sequential algebras, defined relative to BGBI by the Euclidean law

$$
(x \triangleright y) \cdot z \leq x \triangleright(y \cdot z) \text { where } x \triangleright y=\neg(x \backslash \neg y) \text { [JM97]. }
$$

- The variety RA of relation algebras, defined by $x \triangleright y=(x \triangleright 1) y[$ [JT93]. The term $x \triangleright 1$ is the converse operation in relation algebras, denoted by $x^{\smile}$.

- The variety RRA of representable relation algebras, generated by all full relation algebras (cf. $\S 4.1$ ).

- The variety CRA of commutative relation algebras, defined relative to RA by $x y=y x$.

- The variety GRA of group relation algebras, generated by all complex algebras of groups.

- The variety SRA of symmetric relation algebras, defined relative to RA by $x^{\smile=x}$.

- The variety BBI of Boolean BI-algebras (= CRM in [Jip92]).

- LGBI is generated by all linearly ordered GBI-algebras, or equivalently defined by the identity

$$
(x \rightarrow y) \vee(y \rightarrow x)=\top .
$$

- The variety BLBI of basic logic BI-algebras, defined by $x \wedge y=(x / y) \cdot y$.

- The variety HA of Heyting algebras, defined by $x y=x \wedge y$.

- The variety GA of Gödel algebras, defined by $(x \rightarrow y) \vee(y \rightarrow x)=\top$ and $x y=x \wedge y$.

- The variety MVBI of many-valued BI-algebras, defined relative to BLBI by $(x-* \perp)-* \perp=x$.

Figure 5.1 shows how these and some other varieties are related to each other. However, the picture is just a subposet of the infinite lattice of subvarieties of GBI and cannot be used to deduce joins and meets of varieties.

Recall that an algebra is subdirectly irreducible if its congruence lattice has a minimal nontrivial congruence, and that any algebra is a subalgebra of a product of its subdirectly irreducible homomorphic images (cf. [Rafar]). The subdirectly irreducible members of $V$ are denoted by $V_{\mathrm{SI}}$, hence $V=\mathbb{S P}\left(V_{\mathrm{SI}}\right)$.

Every variety $V$ is equal to $\mathbb{V}(\mathfrak{A})$ for some algebra $\mathfrak{A} \in V$ since varieties contain countably generated free algebras. A variety is finitely generated if it is of the form $\mathbb{V}(\mathfrak{A})$ for some finite algebra. In this case, if $\mathbb{V}(\mathfrak{A})$ is congruence distributive (i.e., all members have distributive congruence lattices), then Jónsson's Lemma implies $\mathbb{V}(\mathfrak{A})_{\text {SI }} \subseteq \mathbb{H} \mathbb{S}(\mathfrak{A})$. Hence any subvariety of a finitely generated congruence distributive variety is again finitely generated and for a finite algebra $\mathfrak{A}, \mathbb{V}(\mathfrak{A})$ has only finitely many subvarieties.

\footnotetext{
${ }^{5}$ The reader is encouraged to compare this list with Galatos et al. [GJKO07, $\left.\S 3.5\right]$.
} 


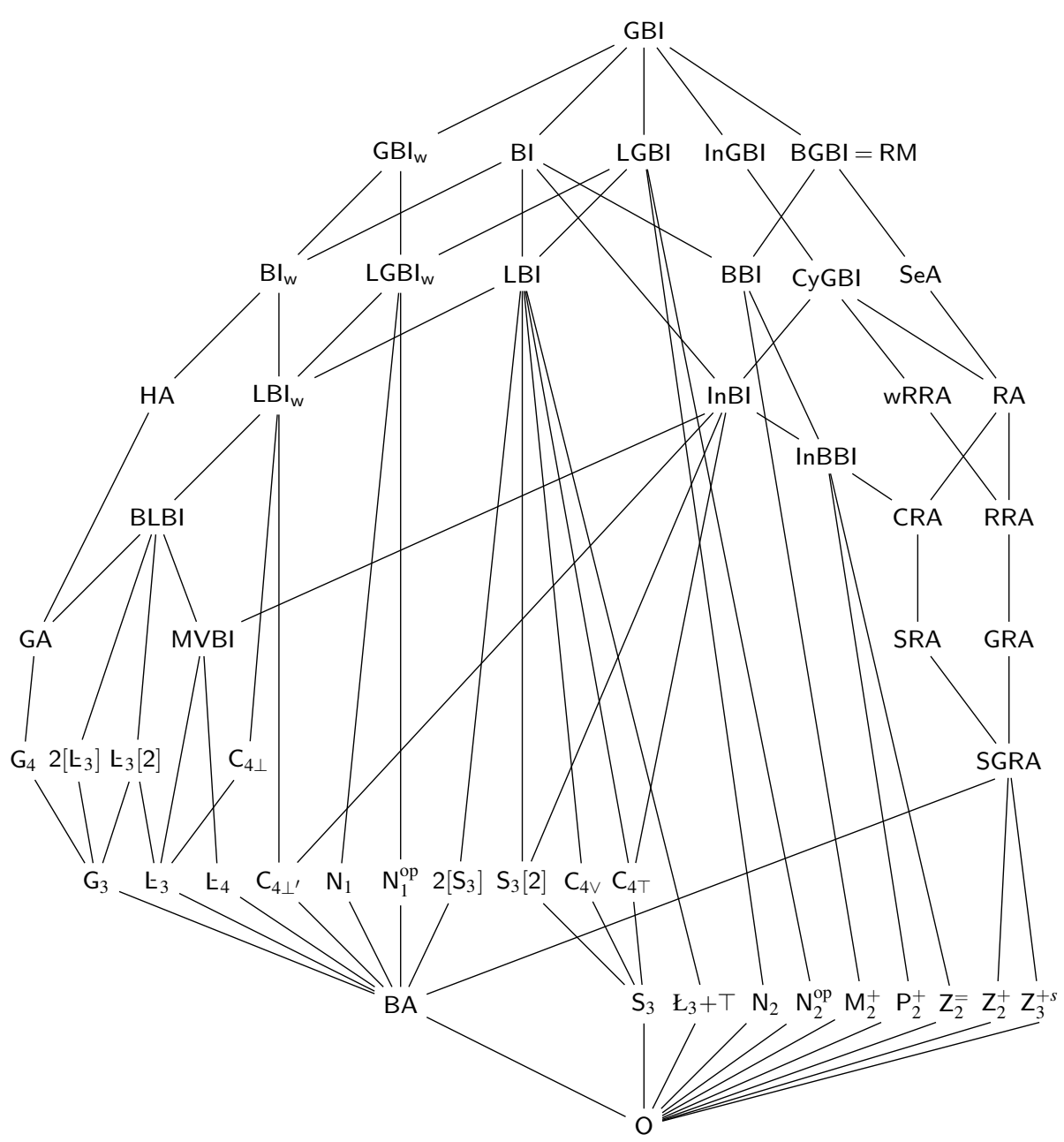

Fig. 5.1 Some subvarieties of GBI ordered by inclusion. Algebras are given in Table 5.1 and denote the variety they generate (in the corresponding font).

Since we also have $\mathbb{V}(\{\mathfrak{A}, \mathfrak{B}\})=\mathbb{V}(\mathfrak{A} \times \mathfrak{B})$, finitely generated subvarieties of $\Lambda$ form a lattice ideal. In particular, the varieties of residuated lattices and GBI-algebras are congruence distributive, since they are varieties of lattice-ordered algebras and all lattices have distributive congruence lattices.

As a result we can investigate the bottom of the lattice $\Lambda$ by investigating finite subdirectly irreducible GBI-algebras. In any GBI-algebra one has $\perp \leq x \backslash \perp$, hence $x \perp=\perp$. It follows that a GBI-algebra with $1=\perp$ must be trivial, hence it generates the variety $\mathrm{O}$. The smallest nontrivial GBI-algebra is the 2-element Boolean algebra, with $1=\top, \cdot=*=\wedge$ and $-*=\rightarrow$. Naturally this algebra generates the variety BA of Boolean algebras.

A 3-element lattice must be linearly ordered, so we can assume $\mathfrak{A}=\{\perp<a<T\}$. There are in fact 3 such algebras: The 3 -element Gödel algebra $\mathfrak{G}_{3}$ where $a * a=a$ and $1=\top$, the 3 -element MV-algebra $\mathfrak{L}_{3}$ where $a * a=\perp$ (hence $1=\top$ ), and the Sugihara algebra $\mathfrak{S}_{3}$ where $a * a=a=1$. The operations $\rightarrow,-*$ are uniquely determined by the order and the monoid operation, and it is easy to check that these algebras are subdirectly irreducible.

An algebra is simple if it has exactly two congruences, and it is strictly simple if, in addition, it has no proper subalgebras. Using Jónsson's Lemma it is easy to see that strictly simple algebras generate varieties that only contain $\mathrm{O}$ as proper subvariety. Note that $\mathfrak{S}_{3}$ is strictly simple, $\mathfrak{L}_{3}$ is simple but not strictly simple, and $\mathfrak{G}_{3}$ is subdirectly irreducible but not simple. Both $\mathfrak{L}_{3}$ and $\mathfrak{G}_{3}$ have a subalgebra isomorphic to the 2-element Boolean algebra, hence they generate varieties with two proper subvarieties. 


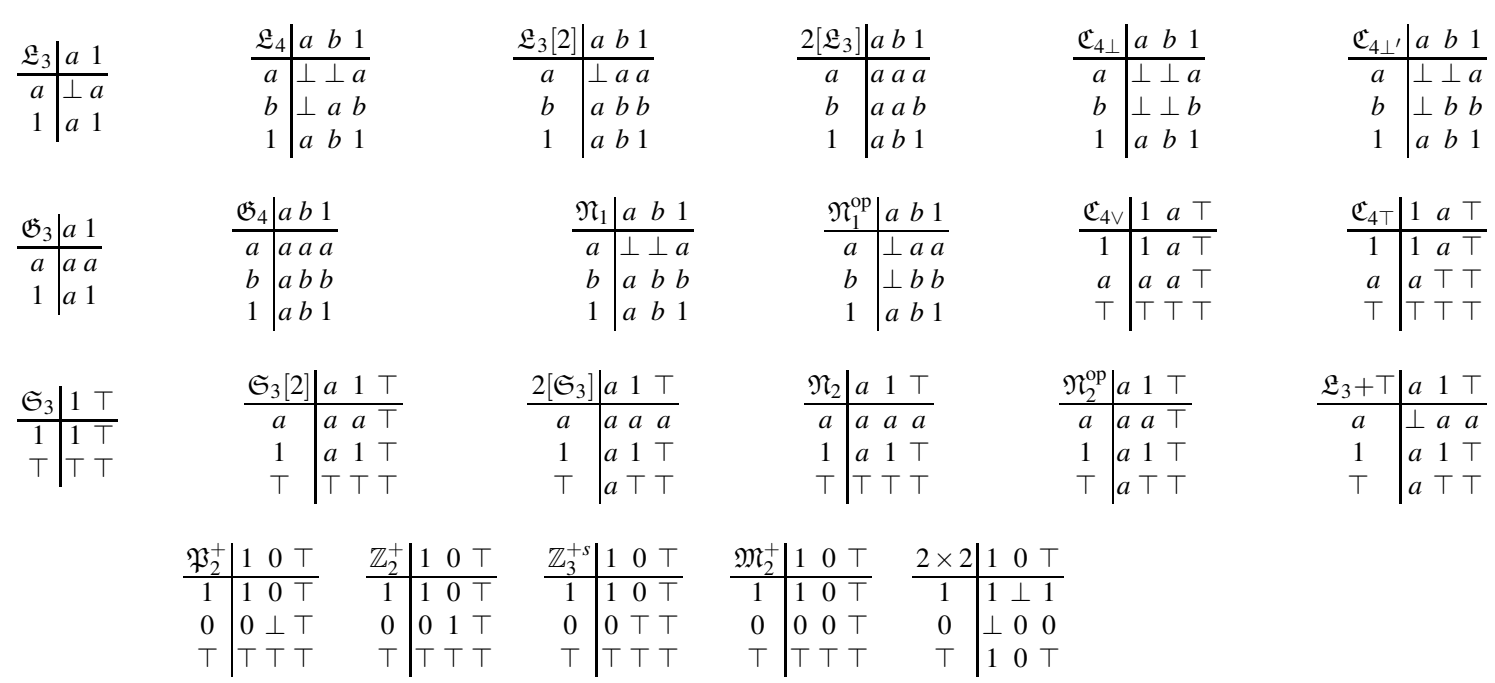

Table 5.1 Multiplicative operation tables for all 3- and 4-element GBI-algebras ( $\perp$ not listed since $\perp x=\perp=x \perp$ ). The order is linear, except for the last row where it is Boolean.

There are several methods for constructing and combining residuated lattices. We consider two that also apply to GBI-algebras. These constructions are used in Table 5.1 to provide convenient names for some of the algebras.

Generalized ordinal sum: This construction, denoted as $\mathfrak{A}[\mathfrak{B}]$ and described in detail by Galatos et al. [GR04; Gal05] [GJKO07, $§ 9.6 .1]$, is applicable with certain restrictions ( $\mathfrak{A}$ must be admissible by $\mathfrak{B}$ ). If $\mathfrak{A}$ satisfies $1=\top$ then this is the usual ordinal sum of two bounded lattice-ordered algebras.

Adding a new top: Let $\mathfrak{A}$ be a GBI-algebra with $1=\top$. The algebra $\mathfrak{A}+\bar{\top}$ is defined by $\mathfrak{A}+\overline{\bar{\top}}=A \cup\{\overline{\mathrm{T}}\}$ where $\bar{\top}$ is strictly greater than $\bar{\top}$. The fusion operation is extended by $\bar{\top} \cdot a=a=a \cdot \bar{\top}$ for all $a \in A-\{1\}$. Hence $\bar{T}$ is almost an identity element, except that $\overline{\bar{T}} 1=\overline{\bar{T}}$. It is easy to check that the operation $\cdot$ is associative, and the residuals are definable in terms of $\cdot$ and $\wedge$. Therefore the algebra $\mathfrak{A}+\bar{\top}$ is also a GBI-algebra.

There are exactly 20 nonisomorphic GBI-algebras with 4 elements (see Table 5.1 and Figure 5.1). The number of nonisomorphic join-preserving monoid operations on a finite distributive lattice increase rapidly:

\begin{tabular}{|c|c|c|c|c|c|c|c|}
\hline$n=$ & 2 & 3 & 4 & 5 & 6 & 7 & 8 \\
\hline GBI & 1 & 3 & 20 & 115 & 899 & 7782 & 80468 \\
BI & 1 & 3 & 16 & 70 & 399 & 2261 & 14358 \\
\hline
\end{tabular}

Any finite distributive residuated lattice is the reduct of a GBI-algebra, but this observation does not extend to the infinite setting. For example, pick a bounded distributive lattice that is not the reduct of a Heyting algebra, and that has an atom 1 . On such a lattice one can define a fusion operation by

$$
x \cdot y= \begin{cases}1 & \text { if } x \neq \perp \neq y \\ \perp & \text { otherwise }\end{cases}
$$

and check that it is a monoid operation that is residuated.

\section{Semantics via Duality}

Heyting algebras and (G)BI-algebras provide algebraic semantics for intuitionistic logic and (noncommutative) bunched implication logic, respectively (cf. $\S 2.3$ and especially Remark 2.3). However the algebras that are of interest can be rather large, or they may have quite complicated order structure. Since they have distributive lattice reducts, it is useful to consider smaller or more concrete combinatorial structures from which the lattice order can be recovered. Considering the categories HA and GBI, with homomorphisms 


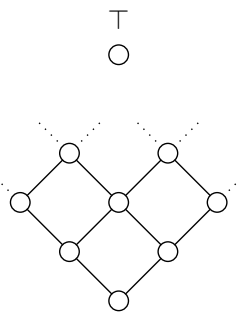

Fig. 6.1 A complete join-perfect distributive lattice that is not meet-perfect. The dual lattice is a Heyting algebra that is not joinperfect.

as morphisms, one would like to have equivalent or dually equivalent categories. For HA there is a welldeveloped duality theory based on Esakia spaces, and we briefly recall the relevant details here. Adding a suitable ternary Kripke relation extends this duality to GBI-algebras as well as to involutive GBI-algebras. Finally we consider a relational semantics based on residuated frames, since this is closely related to the proof theory that we present in $\S 8$.

Before presenting the topological dualities, we first consider Birkhoff's duality for finite distributive lattices, and its extension to complete and perfect distributive lattices. Note that for an element $a$ in a lattice, $\bigvee\{x \mid x<a\}$ always exists, and is either $a$ or a dual cover of $a$. In the latter case, $a$ is said to be completely join-irreducible, and its dual cover is denoted by $a_{*}$. The set of completely join-irreducible elements of a lattice $\mathfrak{L}$ is denoted by $J(\mathfrak{L})$. Dually, a completely meet-irreducible element $b$ satisfies $b \prec$ $\bigwedge\{x \mid b<x\}=b^{*}$, and the set of all such elements is denoted $M(\mathfrak{L})$.

A lattice is complete if all joins and meets exist. Even for a complete lattice, $J(\mathfrak{L})$ and/or $M(\mathfrak{L})$ may be empty, as happens for the unit interval of real numbers.

A lattice is join-perfect if every element is the join of completely join-irreducible elements, it is meetperfect if very element is the meet of completely meet-irreducible elements, and it is perfect if both conditions hold. For example, a Boolean algebra is join-perfect if it is atomic (= every element is a join of atoms, defined as minimal non-zero elements), and every finite lattice is perfect. For a Boolean algebra, being join-perfect is equivalent to being meet-perfect since complementation is a dual isomorphism. However, even for complete distributive lattices this is not the case, as can be seen from the join-perfect distributive lattice $\mathbb{N} \times \mathbb{N}$ completed with a top element, since it has no completely meet-irreducible elements.

Lemma 6.1 Any join-perfect Heyting algebra is also meet-perfect, hence a perfect lattice.

Proof Suppose $a$ is an element in a join-perfect Heyting algebra $\mathfrak{A}$, and let $S=\{x \in M(\mathfrak{A}) \mid a \leq x\}$. If $a \neq \wedge S$ then there exists a lower bound $b$ of $S$ such that $b \not \leq a$. Since $b$ is a join of completely joinirreducibles, there exists $c \in J(\mathfrak{A})$ such that $c \leq b$ and $c \not \leq a$. Let $d=c \rightarrow c_{*}$, where $c_{*}$ is the unique dual cover of $c$. We show that $d \in S$ then it follows that $d \geq b$, which is a contradiction.

From $c \not \leq a$ we get $c \wedge a \leq c_{*}$, hence $a \leq c \rightarrow c_{*}=d$. To see that $d \in M(\mathfrak{A})$, note that if $y>d$ then $y \not \leq c \rightarrow c_{*}$, therefore $c \wedge y \not \leq c_{*}$ and hence $c \leq y$. It follows that $c \leq \wedge\{y \mid d<y\}$, and since $c \not \leq d$ we conclude that $d \prec d^{*}=\bigwedge\{y \mid d<y\}$.

The converse of this lemma does not hold, since for example the dual of $\mathbb{N} \times \mathbb{N}$ with a bottom added is a (complete) meet-perfect Heyting algebra that is not join-perfect.

For complete Heyting algebras, the notion of being perfect is equivalent to being a doubly algebraic lattice (i.e., a complete lattice in which every element is the join of compact elements and the meet of co-compact elements).

\subsection{Semantics and Duality for Heyting Algebras}

Tarski proved that complete and atomic Boolean algebras are isomorphic to powerset algebras, and that complete homomorphisms between Boolean algebras are induced by functions (in the opposite direction) 
between their sets of atoms. In a nutshell this is the categorical duality between caBA, the category of complete and atomic Boolean algebras, and Set, the category of sets.

Birkhoff observed that every finite distributive lattice $\mathfrak{L}$ is isomorphic to the set of downward closed subsets of $(J(\mathfrak{L}), \leq)$, with intersection and union as lattice operations. Alternatively one can take as the starting point the set $F_{\mathrm{p}}(\mathfrak{L})$ of prime filters: in the finite case, $\left(F_{\mathrm{p}}(\mathfrak{L}), \subseteq\right) \cong(J(\mathfrak{L}), \geq)$, hence $\mathfrak{L}$ is isomorphic to the upward closed subsets of $F_{\mathrm{p}}(\mathfrak{L})$.

Actually, it is well known (and easy to see) that for any poset $\mathfrak{P}=(P, \leq)$, the collection $U p(\mathfrak{P})$ of upward closed sets is a complete distributive lattice under $\bigcap$ and $\bigcup$. Since $\bigcup$ distributes over $\cap, U p(\mathfrak{P})$ is in fact a complete Heyting algebra, with $U \rightarrow V=P-\downarrow(U-V)$. The completely join-irreducible elements of $U p(\mathfrak{P})$ are the principal upsets, so $(U p(\mathfrak{P}), \cap, \bigcup)$ is join-perfect (hence perfect) and $\mathfrak{P} \cong J(U p(\mathfrak{P}))$ via the map $p \mapsto \uparrow p$. Likewise, for a complete perfect distributive lattice $\mathfrak{L}, L \cong U p(J(\mathfrak{L}))$. This is a brief outline of the categorical duality between complete and perfect distributive lattices with complete homomorphisms, and posets with order-preserving maps as morphisms. However, as noted earlier $U p(\mathfrak{P})$ is a complete Heyting algebra, and to get a categorical duality, one has to modify the notion of morphism in the semantic category of posets. Rather than allowing all order-preserving maps as morphisms, we restrict to so-called p-morphisms, which are maps such that $\uparrow f(p)=f[\uparrow p]$ for all $p \in P$. (Here $f[X]=\{f(x): x \in X\}$ denotes the forward image of $X$ under $f$. Logicians may note that $\uparrow f(p) \supseteq f[\uparrow p]$ is the "forth" condition, i.e. order preservation, and $\uparrow f(p) \subseteq f[\uparrow p]$ is the "back" condition of modal p-morphisms.)

With this assumption it follows that $f^{-1}[U \rightarrow V]=f^{-1}[U] \rightarrow f^{-1}[V]$ for all $U, V \in U p(\mathfrak{P})$. Hence a p-morphism $f: \mathfrak{P} \rightarrow \mathfrak{Q}$ gives rise to a complete Heyting algebra homomorphism $f^{-1}: U p(\mathfrak{Q}) \rightarrow U p(\mathfrak{P})$. Conversely, a complete Heyting algebra homomorphism $h: \mathfrak{A} \rightarrow \mathfrak{B}$ corresponds to a p-morphism $J(h)$ : $J(\mathfrak{B}) \rightarrow J(\mathfrak{A})$ given by $J(h)(b)=\bigwedge h^{-1}[\uparrow b]$.

In summary, the functors $J: \mathrm{cpHA} \leftrightarrow \mathrm{pPos}: U p$ give the duality between the category of complete perfect Heyting algebras and the category of posets with p-morphisms.

Recall that a Priestley space $(P, \leq, \tau)$ is a poset $(P, \leq)$ with a compact topology $\tau$ on $P$ that has a base of clopen sets and for all $p \not \leq q$ in $P$ there exists a clopen upset $U$ such that $p \in U$ and $q \notin U$. By the wellknown Priestley duality [DP02] the category BDL of bounded distributive lattices with homomorphisms is dually equivalent to the category Pri of Priestley spaces with order-preserving continuous maps.

The functor $F_{\mathrm{p}}: \mathrm{BDL} \rightarrow$ Pri maps a bounded distributive lattice $\mathfrak{L}$ to the Priestley space $\left(F_{\mathrm{p}}(\mathfrak{L}), \subseteq, \tau\right)$. As before, $F_{\mathrm{p}}(\mathfrak{L})$ is the set of all prime filters of $\mathfrak{L}$, ordered by inclusion, and the topology $\tau$ is generated by the subbasis $\left\{\mathscr{F}_{a}: a \in L\right\} \cup\left\{F_{\mathrm{p}}(\mathfrak{L})-\mathscr{F}_{a}: a \in L\right\}$ where $\mathscr{F}_{a}=\left\{F \in F_{\mathrm{p}}(\mathfrak{L}): a \in F\right\}$. For a homomorphisms $h: L \rightarrow M$, the map $F_{\mathrm{p}}(h): F_{\mathrm{p}}(M) \rightarrow F_{\mathrm{p}}(\mathfrak{L})$ given by $F_{\mathrm{p}}(h)(G)=h^{-1}[G]$ is a continuous and order-preserving.

The functor $U_{\mathrm{c}}:$ Pri $\rightarrow$ BDL maps a Priestley space $\mathfrak{P}=(P, \leq, \tau)$ to the set $U_{\mathrm{c}}(\mathfrak{P})$ of clopen upsets, which is a bounded distributive lattice under intersection and union. For a continuous order-preserving map $f:(P, \leq, \tau) \rightarrow\left(Q, \leq, \tau^{\prime}\right)$, the map $U_{\mathrm{c}}(f): U_{\mathrm{c}}(\mathfrak{Q}) \rightarrow U_{\mathrm{c}}(\mathfrak{P})$ given by $U_{\mathrm{c}}(f)(V)=f^{-1}[V]$ is a bounded distributive lattice homomorphism.

An Esakia space is a Priestley space $(P, \leq, \tau)$ such that if $U \subseteq P$ is clopen then $\downarrow U$ is also clopen. The restriction of Priestley duality to Heyting algebras is given by the following result.

\section{Theorem 6.2}

- A bounded distributive lattice $\mathfrak{L}$ is a Heyting algebra if and only if the corresponding Priestley space $F_{p}(\mathfrak{L})$ is an Esakia space.

- For Heyting algebras $\mathfrak{A}, \mathfrak{B}$ a bounded distributive lattice homomorphism $h: A \rightarrow B$ preserves the Heyting implication if and only if $F_{p}(h)$ is a p-morphism.

\subsection{Semantics and Duality for GBI-algebras}

To extend this duality to GBI-algebras, we need a ternary relation $\circ: P^{2} \rightarrow \mathscr{P}(P)$ and a unary relation $E \subseteq P$ satisfying certain conditions to an Esakia space $(P, \leq, \tau)$. The definitions and results of this subsection are based on Urquhart [Ura96] and Galatos [Gal00]; cf. also more recent references motivated by CS applications of BI [DP17a; DP17b; BV14], where similar dualities or representation theorems have been 
developed independently. We extend $\circ$ to a binary operation on $\mathscr{P}(P)$ by

$$
X \circ Y=\bigcup_{x \in X, y \in Y} x \circ y, \quad x \circ Y=\{x\} \circ Y, \quad X \circ y=X \circ\{y\}
$$

and let

$$
X \cdot Y=\uparrow(X \circ Y), X \backslash Y=\{z: X \circ z \subseteq Y\}, X / Y=\{z: z \circ Y \subseteq X\} .
$$

Now define $\mathfrak{P}=(P, \leq, \circ, E, \tau)$ to be a $G B I$-space if $(P, \leq, \tau)$ is an Esakia space and the following properties hold:

1. $(x \circ y) \circ z=x \circ(y \circ z)$,

2. $E$ is a clopen upset, and $E \circ U=U=U \circ E$ for any clopen upset $U$,

3. $x^{\prime} \leq x, y^{\prime} \leq y, z \leq z^{\prime}$, and $z \in x \circ y \Longrightarrow z^{\prime} \in x^{\prime} \circ y^{\prime}$,

4. for all clopen upsets $U, V$ of $P, U \circ V, U \backslash V$ and $U / V$ are clopen and

5. $z \notin x \circ y$ implies there exist clopen upsets $U, V$ such that $x \in U, y \in V$ and $z \notin U \circ V$.

For GBI-spaces $\mathfrak{P}, \mathfrak{Q}$ a $G B I$-p-morphism $f: \mathfrak{P} \rightarrow \mathfrak{Q}$ is a continuous Heyting algebra p-morphism $f:(P, \leq$ $, \tau) \rightarrow\left(Q, \leq, \tau^{\prime}\right)$ that satisfies 6

6. $z \in x \circ_{\mathfrak{P}} y \Longrightarrow f(z) \in f(x) \circ_{\mathfrak{Q}} f(y)$,

7. $f(z) \in u \circ \mathfrak{Q} v \Longrightarrow \exists x, y \in P(u \leq f(x), v \leq f(y)$ and $z \in x \circ \mathfrak{P} y)$,

8. $w \in f(x) \circ_{\mathfrak{Q}} v \Longrightarrow \exists y, z \in P(v \leq f(y), f(z) \leq w$ and $z \in x \circ \mathfrak{P} y)$,

9. $w \in u \circ_{\mathfrak{Q}} f(y) \Longrightarrow \exists x, z \in P\left(u \leq f(x), f(z) \leq w\right.$ and $\left.z \in x \circ_{\mathfrak{P}} y\right)$, and

10. $E_{\mathfrak{P}}=f^{-1}\left[E_{\mathfrak{Q}}\right]$.

The category of GBI-spaces with GBI-p-morphisms is denoted by GBIp. Finally we define the functor $F_{\mathrm{p}}: \mathrm{GBI} \rightarrow \mathrm{GBlp}$ by

$$
F_{\mathrm{p}}(\mathfrak{A})=\left(F_{\mathrm{p}}(A), \subseteq, \circ, \mathscr{F}_{1}, \tau\right)
$$

where

- $\circ: F_{\mathrm{p}}(A)^{2} \rightarrow \mathscr{P}\left(F_{\mathrm{p}}(A)\right)$ is given by $F \circ G=\bigcap_{a \in F, b \in G} \mathscr{F}_{a b}$ and

- $\left(F_{\mathrm{p}}(A), \subseteq, \tau\right)$ is the Esakia space of the Heyting algebra reduct of $\mathfrak{A}$.

Conversely, the functor $U_{\mathrm{c}}: \mathrm{GBIp} \rightarrow \mathrm{GBI}$ is defined by

$$
U_{\mathrm{c}}(\mathfrak{P})=\left(U_{\mathrm{c}}(P), \cap, \cup, \rightarrow, \emptyset, P, \cdot, \backslash, /, E\right) .
$$

On morphisms, these two functors act the same way as for Heyting algebras and Esakia spaces.

\section{Theorem 6.3}

(i) For any GBI-algebra $\mathfrak{A}, F_{\mathrm{p}}(\mathfrak{A})$ is a GBI-space.

(ii) For any GBI-space $\mathfrak{P}, U_{\mathrm{c}}(\mathfrak{P})$ is a GBI-algebra.

(iii) $U_{\mathrm{c}}\left(F_{\mathrm{p}}(\mathfrak{A})\right) \cong \mathfrak{A}$ and $F_{\mathrm{p}}\left(U_{\mathrm{c}}(\mathfrak{P})\right) \cong \mathfrak{P}$.

(iv) On morphisms, the functors $U_{\mathrm{c}} F_{\mathrm{p}}$ and $F_{\mathrm{p}} U_{\mathrm{c}}$ are naturally isomorphic to the respective identity functors, hence the category of GBI-algebras is dually equivalent to the category of GBI-spaces.

Proof Since the details of Priestley and Esakia duality are well known [DP02; DG03], we verify only the properties related to $\cdot, \backslash, /, 1, \circ$ and $E$. We first state some auxiliary facts that are easy to check. For filters $F, G$ of $\mathfrak{A}$ we let $F G=\{a b: a \in F, b \in G\}$.

Claim 1. $\uparrow(F G)$ is a filter of $\mathfrak{A}$. Pf: For any $a, b \in \uparrow(F G)$ there exist $a^{\prime}, b^{\prime} \in F, a^{\prime \prime}, b^{\prime \prime} \in G$ such that $a^{\prime} a^{\prime \prime} \leq a$ and $b^{\prime} b^{\prime \prime} \leq b$. Hence $a^{\prime} \wedge b^{\prime} \in F, a^{\prime \prime} \wedge b^{\prime \prime} \in G$ and $a \wedge b \geq a^{\prime} a^{\prime \prime} \wedge b^{\prime} b^{\prime \prime} \geq\left(a^{\prime} \wedge b^{\prime}\right)\left(a^{\prime \prime} \wedge b^{\prime \prime}\right) \in F G$.

Claim 2. For $F, G, H \in F_{\mathrm{p}}(A), H \in F \circ G$ if and only if $F G \subseteq H$. Pf: Follows from the definition $F \circ G=$ $\bigcap_{a \in F, b \in G} \mathscr{F}_{a b}$.

Claim 3. For filters $F, G$ of $\mathfrak{A}$ and $F G \subseteq H \in F_{\mathrm{p}}(A)$, there exist $F^{\prime}, G^{\prime} \in F_{\mathrm{p}}(A)$ such that $F^{\prime} G, F G^{\prime}, F^{\prime} G^{\prime} \subseteq H$.

\footnotetext{
${ }^{6}$ The need for items 8. and 9. was pointed out by Docherty and Pym, see [DP17a; DP17b]. Here we use item 8. as first introduced by Urquhart [Ura96] for relevant implication $x \rightarrow y=y / x$, and item 9. is the corresponding version for $\backslash$.
} 
(i) We prove properties 1.-5. of GBI-spaces. For 1. let $F, G, H, K \in F_{\mathrm{p}}(A)$ and assume $K \in(F \circ G) \circ H$. Then there exists $M \in F \circ G$ such that $K \in M \circ H$. By Fact 2, $F G \subseteq M$ and $M H \subseteq K$. Consider the filter $N=\uparrow(G H)$ and note that $F N \subseteq K$ since $\cdot$ is associative. By Fact 3, there exists $N^{\prime} \in F_{\mathrm{p}}(A)$ such that $N \subseteq N^{\prime}$ and $F N^{\prime} \subseteq K$. Since we also have $G H \subseteq N^{\prime}$ it follows that $K \in F \circ N^{\prime}$ and $N^{\prime} \in G \circ H$, whence $K \in F \circ(G \circ H)$. The converse is similar.

Recall that $\mathscr{F}_{1}=\left\{F \in F_{\mathrm{p}}(A): 1 \in F\right\}$, which is a subbasic clopen of $\tau$ and is also an upset in the inclusion order on prime filters. Let $\mathscr{U}$ be a clopen upset of prime filters. By Priestley duality $\mathscr{U}=\mathscr{F}_{a}$ for some $a \in A$, hence $\mathscr{F}_{1} \circ \mathscr{U}=\mathscr{F}_{1} \circ \mathscr{F}_{a}=\mathscr{F}_{1 a}=\mathscr{F}_{a}=\mathscr{U}$. The other equality of 2 is similar.

For 3. let $F^{\prime} \subseteq F, G^{\prime} \subseteq G, H \subseteq H^{\prime}$ and $H \in F \circ G$. Then $F G \subseteq H \subseteq H^{\prime}$ so $F^{\prime} G^{\prime} \subseteq F G \subseteq H^{\prime}$ which implies $H^{\prime} \in F^{\prime} \circ G^{\prime}$.

Let $\mathscr{U}, \mathscr{V}$ be clopen upsets of prime filters. By Priestley duality there exist $a, b \in A$ such that $\mathscr{U}=\mathscr{F}_{a}$ and $\mathscr{V}=\mathscr{F}_{b}$. We prove that $\mathscr{F}_{a} \circ \mathscr{F}_{b}=\mathscr{F}_{a b}, \mathscr{F}_{a} \backslash \mathscr{F}_{b}=\mathscr{F}_{a \backslash b}$ and $\mathscr{F}_{a} / \mathscr{F}_{b}=\mathscr{F}_{a} / b$, then 4. holds. Let $H \in \mathscr{F}_{a} \circ \mathscr{F}_{b}$, so $H \in F \circ G$ where $a \in F$ and $b \in G$ for prime filters $F, G$. Then $a b \in F G \subseteq H$ by Claim 2, whence $H \in \mathscr{F}_{a b}$. Conversely, if $H \in \mathscr{F}_{a b}$ then $\uparrow a \uparrow b \subseteq H$. By Claim 3 there exist prime filters $F, G$ such that $\uparrow a \subseteq F, \uparrow b \subseteq G$ and $F G \subseteq H$, so $H \in F \circ G \subseteq \mathscr{F}_{a} \circ \mathscr{F}_{b}$.

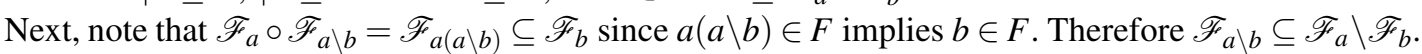
For the opposite inclusion, let $G \in \mathscr{F}_{a} \backslash \mathscr{F}_{b}$ whence $\mathscr{F}_{a} \circ G \subseteq \mathscr{F}_{b}$. We aim to show that $G \in \mathscr{F}_{a} b$, i.e., $a \backslash b \in G$. If $a=\perp$ this holds trivially, so assume $a \neq \perp$ and let $F \in \mathscr{F}_{a}$. Then for any $H \in F \circ G$ we have $H \in \mathscr{F}_{b}$, or equivalently, $F G \subseteq H$ implies $b \in H$. By Claim $1, \uparrow(F G)$ is a filter, so $b \in \uparrow(F G)$ (otherwise we could extend this to a prime filter $H$ that does not contain $b$ ). We conclude that $a x \leq b$ for some $x \in G$, whence $x \leq a \backslash b \in G$. The argument for $a / b$ is similar.

Finally, for 5. let $F, G, H$ be prime filters such that $H \notin F \circ G$. Then $\uparrow(F G) \not \subset H$, so there exist $a \in F, b \in$ $G$ such that $a b \leq c \notin H$. Then $F \in \mathscr{F}_{a}, G \in \mathscr{F}_{b}$ and $\mathscr{F}_{a} \circ \mathscr{F}_{b}=\mathscr{F}_{a b}$, hence $H \notin \mathscr{F}_{a} \circ \mathscr{F}_{b}$.

(ii) For a GBI-space $\mathfrak{P}$, we take for granted that $\left(U_{\mathrm{c}}(P), \cap, \cup, \rightarrow, \top, \perp\right)$ is a Heyting algebra. By Property 1 the ternary relation $\circ$ lifts to an associative operation on $\mathscr{P}(P)$. By Property 2 the set $E$ is a unit element for this lifted operation when restricted to clopen upsets. For $U, V \in U_{\mathrm{c}}(P)$ Property 3 implies that $U \circ V$ is also an upset, and by Property 4 it will be clopen. Hence $\left(U_{\mathrm{c}}(P), \circ, E\right)$ is a monoid.

By definition $x \in U / V \Longleftrightarrow x \circ V \subseteq U$, and for $x \leq w$ we have $w \circ V \subseteq x \circ V$ since $z \in w \circ V$ implies $z \in w \circ y$ for some $y \in V$, so $z \in x \circ y$ by Property 3. Therefore $w \circ V \subseteq U$, or equivalently $w \in U / V$, which shows that $U / V$ is an upset. By Property 4 it is also clopen, hence $U / V \in U_{\mathrm{c}}(P)$. The argument for $U \backslash V$ is similar.

Now suppose $W \subseteq U / V$. This is equivalent to $w \circ V \subseteq U$ for all $w \in W$ which in turn is equivalent to $W \circ V \subseteq U$. Hence $U_{\mathrm{c}}(\mathfrak{P})$ is a GBI-algebra.

(iii) By Priestley duality, the map $a \mapsto \mathscr{F}_{a}$ is a bounded distributive lattice isomorphism from $\mathfrak{A}$ to $U_{\mathrm{c}}\left(F_{\mathrm{p}}(\mathfrak{A})\right)$, and since $\rightarrow$ is uniquely determined by the lattice, it is also a Heyting algebra isomorphism. Furthermore, by the proof of Property 4 in (i) this map preserves $\circ, \backslash$, and $/$. For a GBI-space $\mathfrak{P}$ consider the map $f: P \rightarrow F_{\mathrm{p}}\left(U_{\mathrm{c}}(\mathfrak{P})\right.$ defined by $f(x)=\left\{U \in U_{\mathrm{c}}(P): x \in U\right\}$. This is an isomorphism of the Esakia spaces and it suffices to check that $z \in x \circ y$ if and only if $f(z) \in f(x) \circ f(y)$.

(iv) It remains to check that the functor $F_{\mathrm{p}}$ sends a GBI homomorphisms to a GBI-p-morphism, and the functor $U_{\mathrm{c}}$ does the reverse. For details of this argument we refer the reader to Urquhart [Urq96] (Thm 3.5). The categorical duality then follows by restricting the duality for Heyting algebras and Esakia spaces to GBI-homomorphisms and GBI-p-morphisms.

\section{Decidability Issues}

\subsection{Positive Decidability Results}

Let us begin with a result that can be derived from the proof-theoretic framework described in Section 8 and developed by the first author in collaboration with Nick Galatos; see the discussion therein (also for related references like [Koz09]). Let nGBI be the variety of nonassociative GBI-algebras, i.e., defined by the axioms of GBI but without the axiom of associativity of fusion; see, e.g., Galatos and Ono [GO10] or Galatos et al. [GJKO07, Ch. 2.3.1] for more information on non-associative substructural logics (cf. also Rem.7.5 and [DP17a]). 
Theorem 7.1 The equational theory of $\mathrm{nGBI}$ and $\mathrm{BI}$ is decidable.

Decidability of $\mathrm{BI}$ is proved by Galmiche et al. [GMP05]. The proof in that paper uses specific, tailormade techniques. We are not aware of any reference prior to Galatos and Jipsen [GJ17] claiming decidability for $\mathrm{nGBI}$ or GBI.7

It seems that at the moment, there is no systematic investigation of complexity of these equational theories; some upper bounds are claimed by Ramanayake [Ram16]. Another problem which seems presently open is the question of decidability of non-boolean involutive varieties like $\operatorname{lnGBI}$ and $\mathrm{CyGBI}$.

Obviously, there are subareas of $\Lambda$ which allow nice decidability results for whole classes of varieties, in fact going beyond purely equational theory. Apart from finitely generated varieties like BA and other ones in the bottom area of Figure 5.1 we have numerous decidability results for subvarieties of HA. In fact, many of them enjoy rather low complexity, from PSPACE for HA itself to NP for GA and its subvarieties. These results are well described in standard monographs [CZ97].

However, in other parts of $\Lambda$ positive decidability results are much less common. Moreover, things look even worse when one goes beyond purely equational theory. Let us discuss the two issues separately.

\subsection{Subvarieties with Undecidable Equational Theory}

As it turns out, undecidability results abound for BGBI (also known as RM) and its extensions, in particular BBI. Powerful general undecidability results for such varieties were established by algebraic logicians in the 1990's and summarized in references like Andréka et al. [AKNSS96] or earlier Kurucz et al. [KNSS95]. Here is a result most relevant for our purposes:

Theorem 7.2 A variety $V \subseteq \mathrm{BGBI}$ is undecidable whenever

- there exists an infinite algebra $\mathfrak{A} \in V$ containing a $(\cdot, \backslash, /)$-subreduct whose universe is an antichain in $\mathfrak{A}$ or

- every finite group is semigroup-embeddable into some $\mathfrak{A} \in V$ or

- for any $n \in \mathbb{N}$ there exists a product $\mathfrak{G}$ of n nontrivial finite groups and a semigroup-embedding $f: G \rightarrow$ A into (the semigroup-reduct of) some $\mathfrak{A} \in V$ s.t. $f[G]$ is an antichain in $\mathfrak{A}$.

Proof Follows from Kurucz et al. [KNSS95, Th. 8], [AKNSS96, Th. 3.6].

Corollary 7.3 Equational theories of $\mathrm{BGBI}=\mathrm{RM}, \mathrm{BBI}=\mathrm{CRM}, \mathrm{IRM}, \mathrm{RA}, \mathrm{ICRM}, \mathrm{IRA}, \mathrm{CRA}, \mathrm{RRA}, \mathrm{GRA}$, SRA and SGRA are undecidable.

For most of these classes, this corollary is explicitly stated as Corollary 5.4 in Andréka et al. [AKNSS96] or Corollary 8.1 in Kurucz et al. [KNSS95]. Of course, for varieties like RA and RRA undecidability of the equational theory was established much earlier by Tarski, in fact claimed already in 1941 [Tar41].

These results were somehow overlooked by the BI community. Much more recently, overlapping undecidability results for subvarieties of BBI have been obtained by Brotherston and Kanovich [BK10; BK14] and by Larchey-Wendling and Galmiche [LG10; LG13]. However, we repeatedly stated in $\S 1$ and $\S \S 34$ that it is natural to focus on concrete models, particularly memory and heap models ( $\$ 3.4$ ). Brotherston and Kanovich [BK14] prove that subvarieties of BBI generated by such models are undecidable; similar undecidability results apply to even the simplest allocation/effect algebras (cf. $\S 3.3$ ). An alternative, somewhat more semantic proof is provided by Demri and Deters [DD15a, Theorem 4.4]. It is not immediately clear how to adjust algebraic proofs quoted above to cover such varieties: Andréka et al. [AKNSS96] follow Urquhart [Urq84; Urq95], Freese [Fre80] and Lipshitz [Lip74] in using the technique of von Neumann's [Neu60] $n$-frames, which originated in projective geometries and is applicable to a wide class of varieties, but rather heavy on the technical side. In contrast, Brotherston and Kanovich [BK14] or Demri and Deters [DD15a] use a natural strategy of encoding Minsky machines, tailored for the intended models, and more readily understandable to CS researchers.

\footnotetext{
${ }^{7}$ The proof of decidability of GBI in Galatos and Jipsen [GJ17] appears to have an issue with the complexity measure, as pointed out by R. Ramanayake. Decidability of nGBI has also been proved by Docherty and Pym [DP17a], independently of the same result in Galatos and Jipsen [GJ17.
} 
Remark 7.4 It is important to mention here that the separation logic community not only tends to be interested in concrete models, but also typically extends the syntax with entities allowing reasoning about e.g. heap structure and program values. Moreover, BI and its extensions are not considered in isolation, but are of interest mostly as the assertional core of proper separation logic, i.e., a suitable language of Hoare triples $(\S 9$. On the other hand, the assertion language hardly ever allows uninterpreted algebraic variables, which also limits direct applicability of (un)decidability results discussed here. Demri and Deters [DD15a] provide an overview of positive and negative results for the assertion language of separation logic. We will return to the issue in $\S 10.2$

Remark 7.5 Finally, let us note that these undecidability results heavily rely on associativity. The concluding section of Andréka et al. [AKNSS96] or, even more relevantly, the chapter by Mikulás [Mik96] in the same volume [MPM96] show that positive decidability results are available even for boolean nGBI and its relatives. An explanation of practical interest in such formalisms can be found in the recent work of Collinson et al. [CMP14]; in fact, this reference rediscovers a variant of system called CARL by Mikulás [Mik96]. The intuitionistic variant (in fact equivalent to the equational theory of nGBI [GJ17]; cf. also [GO10], [GJKO07, Ch. 2.3.1]) is motivated in a similar setting by Docherty and Pym [DP17b].

\subsection{Undecidability of Quasi-Equational Theories}

Obviously, all the subvarieties with undecidable equational theory discussed in $\S 7.2$ have a fortiori undecidable quasi-equational theories. Nevetheless, even having decidable equational theory does not guarantee positive results here (see, however, Remark 8.6).

Theorem 7.6 The quasi-equational theory of $\mathrm{BI}$ is undecidable.

Proof (Sketch) Galatos [Gal02], following a strategy similar to that of Urquhart [Urq84; Urq95], Freese [Fre80] and, earlier still, of Lipshitz [Lip74] shows that the word problem for DRL (distributive residuated lattices, cf. [GJKO07, $\S 3.5 .3$ ]) is undecidable. As will be shown in $\S$, this class consist precisely of subreducts of $\mathrm{BI}$ which implies the result.

Remark 7.7 The same technique can be used to show directly the undecidability of the quasiequational theory of BBI: just replace DRL with CDRL (commutative distributive residuated lattices; $\mathrm{cf}$. $\S 8$ ). But in the boolean setting, Corollary 7.3 provides a stronger result anyway. The general idea of using von Neumann's $n$-frames is central to both proofs.

Recall that the finite embeddability property (cf., e.g., [BV02]) for finitely axiomatizable (quasi)varieties implies decidability of universal theory - and, a fortiori, quasi-equational theory. Thus we obtain

Corollary 7.8 GBI and $\mathrm{BI}$ do not have the finite embeddability property.

For BBI, we have an even stronger result that follows from Corollary 7.3

Corollary 7.9 $\mathrm{BBI}$ does not have the finite model property.

Still, as pointed out in Remark 8.6, the f.e.p. often holds in the presence of weakening. This includes in particular the intuitionistic logic, and it is worth pointing out here Recall that the finite embeddability property for HA was already noted in a classical paper of McKinsey and Tarski [MT46, Th. 1.11].

\section{A Glimpse at Proof Theory}

The formulas of GBI are all terms constructed from variables $x, y, z, w, x_{1}, \ldots$ using the operation symbols $\wedge, \vee, \rightarrow, \top, \perp, \cdot, \backslash, /, 1$. The set of all these terms is the absolutely free term algebra of this signature, denoted by $\mathfrak{F m}_{\mathrm{GBI}}$. In this section we give a syntactic sequent calculus that provides a decision procedure for testing if an inequality $s \leq t$ holds in all GBI-algebras. The proof that the procedure is complete uses the notion of distributive residuated frame and algebraic cut-elimination, due to Galatos and Jipsen [GJ13; GJ17]. 


$$
\begin{gathered}
\overline{x \leq x} \quad \overline{u(\perp) \leq x} \quad \overline{x \leq \top} \\
\frac{u(x \wedge x) \leq y}{u(x) \leq y}\left[\wedge_{i d e m}\right] \quad \frac{u(x) \leq z}{u(x \wedge y) \leq z}\left[\wedge_{l}\right] \quad \frac{u(y) \leq z}{u(x \wedge y) \leq z}\left[\wedge_{l}\right] \quad \frac{x \leq y \quad x \leq z}{x \leq y \wedge z}\left[\wedge_{r}\right] \\
\frac{u(x) \leq z \quad u(y) \leq z}{u(x \vee y) \leq z}\left[\vee_{l}\right] \quad \frac{x \leq y}{x \leq y \vee z}\left[\vee_{r}\right] \quad \frac{x \leq z}{x \leq y \vee z}\left[\vee_{r}\right] \\
\frac{x \leq y \quad u(z) \leq w}{u(x \cdot(y \backslash z)) \leq w}[\backslash l] \quad \frac{x \cdot y \leq z}{y \leq x \backslash z}[\backslash r] \quad \frac{x \leq y}{u((z / y) \cdot x) \leq w} \quad u(z) \leq w \\
\frac{x \leq y \quad z \leq w}{x \cdot z \leq y \cdot w}\left[\cdot l_{l r}\right] \quad \frac{x \cdot y \leq z}{x \leq z / y}[/ r] \\
\frac{x \leq y \quad u(z) \leq w}{u(x \wedge(y \rightarrow z)) \leq w}[\rightarrow l] \quad \frac{x \wedge y \leq z}{y \leq x \rightarrow z}\left[\rightarrow_{r}\right]
\end{gathered}
$$

Table 8.1 The sequent rules of GBI.

The equational decidability of the distributive residuated lattice reduct is also proved by Kozak [Koz09]. Of course, especially in this overview we have to recall that the technique of algebraic cut-elimination dates back to Belardinelli, Jipsen and Ono [BJO04] (see also [GJKO07, Ch. 7], [GO10]).

The approach outlined here works for all subvarieties of GBI that are defined by so-called simple structural rules, which includes $\mathrm{BI}$ and many others. In addition to proving decidability, the residuated frame approach also provides a completion that shows any (commutative) distributive lattice-ordered monoid can be embedded in a complete GBI-algebra (BI-algebra) 8

We use an algebraic approach for the sequent calculus, allowing rules like associativity of $\cdot, \wedge$ to be handled by a simple normalization of terms. This means that we replace the algebra $\mathfrak{F m}_{\mathrm{GBI}}$ by a homomorphic image in which terms are identified modulo associativity for - and modulo commutativity and associativity for $\wedge$. This is harmless since each term has only finitely many equivalent syntactic forms modulo these identities. In addition a formula $x$ can be considered as any one of

$$
x=x \cdot 1=1 \cdot x=x \wedge \top=\top \wedge x=x \vee \perp=\perp \vee x
$$

when attempting to match a sequent rule. To avoid proliferation of the sequent rules, we also do not distinguish between internal and external symbols of the sequent calculus, but we define a notion of context (bunches) that handles the required constraint automatically.

In the sequent rules (quasiequations) below, the bunches $u(x)$ are terms in which the variable $x$ occurs only once, and on the term-tree branch where $x$ occurs, only the symbols · and $\wedge$ are allowed to appear.

Lemma 8.1 The rules in Table 8.1 are valid quasiequations of GBI.

Proof (Sketch) In any GBI-algebra, $u(x)$ induces an order-preserving term-function of $x$ under any assignment of the other variables in $u(x)$.

The effectiveness of these quasiequations stems from the observation that each rule contains the same variables in the premise and in the conclusion, and each rule (except $\wedge_{\text {idem }}$ ) eliminates a particular symbol either from the left hand side or the right hand side of the conclusion, as indicated by its name. When the rules are applied in a backward proof search, the conclusion is matched to the inequality $s \leq t$, and this match determines the assignments to the variables in the premise. Furthermore, the premises of each rule (again, except $\wedge_{\text {idem }}$ discussed below) are at most as long as the conclusion (using some standard measure of length of a formula). Hence symbols get eliminated from $s, t$ as the search proceeds, and the inequalities in the premises do not grow in length, so after a finite number of steps the search either terminates with

\footnotetext{
${ }^{8}$ Needless to say, the residuated frame approach to proof theory of $(\mathrm{G}) \mathrm{BI}$ is not the only possible one. As we have already pointed out, there is an intimate connection with a massive body of work on proof theory of relevance logics. See comments and references in the Introduction. We should also mention here that there are numerous more recent references, e.g., cut-free proof calculi for subvarieties of $\mathrm{BI}$ of Ciabattoni and Ramanayake [CR17].
} 
axioms as leaves, or having exhausted all possible applications of the rules the conclusion is that $s \leq t$ cannot be proved by this sequent calculus.

In the remainder of this section we outline why this proof procedure yields all valid inequalities of $\mathrm{GBI}$, and how it extends to cover many of its subvarieties.

The following quasiequation, known as the cut-rule, does not have the subformula property:

$$
\frac{x \leq y \quad u(y) \leq z}{u(x) \leq z}[\mathrm{cut}]
$$

Adding this rule to the GBI sequent calculus makes it quite easy to show that sequent proofs (with cut) can emulate Hilbert system proofs in HGBI, and hence the sequent calculus is complete with respect to the equational theory of GBI. E.g., the cut rule emulates modus ponens in the form $\frac{T \leq y \quad y \leq z}{\top \leq z}$ and a proof of the axiom $x \rightarrow(y \rightarrow x)$ is given by

$$
\frac{\frac{x \leq x}{x \wedge y \leq x}}{\frac{x \leq y \rightarrow x}{x \wedge \top \leq y \rightarrow x}} \frac{}{\top \leq x \rightarrow(y \rightarrow x) .}
$$

Nevertheless, the cut-rule cannot be used effectively in a proof search, since the variable $y$ in the premise can be instantiated with any formula, hence the search tree is not finite. One, however, can show that the GBI-calculus without the cut-rule is able to prove exactly the same inequalities as can be proved with the cut-rule. There are several approaches to proving such a cut-elimination result, but with our emphasis on algebraic aspects of $(\mathrm{G}) \mathrm{BI}$ we choose to outline an algebraic approach based on Galatos and Jipsen [GJ17].

A binary relation $N$ from a set $W$ to $W^{\prime}$ is a map $N: W \rightarrow \mathscr{P}\left(W^{\prime}\right)$ and a ternary relation $\circ$ on $W$ is a map $\circ: W \times W \rightarrow \mathscr{P}(W)$. Instead of $z \in N(x)$ we write $x N z$, and for sets $X, Y \subseteq W, Z \subseteq W^{\prime}$ define

- $X N z$ iff $x N z$ for all $x \in X$,

- $x N Z$ iff $x N z$ for all $z \in Z$,

$-x \circ y=\circ(x, y)$,

- $X \circ Y=\bigcup\{x \circ y: x \in X, y \in Y\}$ and

- $\gamma: \mathscr{P}(W) \rightarrow \mathscr{P}(W)$ by $\gamma(X)=\{z \in W: \forall y(X N y$ implies $z N y)\}$.

Note that $\gamma$ is a closure operator on $W$, i.e., $X \subseteq \gamma(X)=\gamma(\gamma(X))$.

A GBI-frame is a structure $\mathfrak{W}=\left(W, W^{\prime}, N, \circ, E, \| \backslash, / /, \curlywedge, \Rightarrow, \Leftarrow\right)$, where $N \subseteq W \times W^{\prime}, \circ, \curlywedge$ are ternary relations on $W$,

$$
\left\|, \Rightarrow: W \times W^{\prime} \rightarrow \mathscr{P}(W) \quad\right\|, \Leftarrow: W^{\prime} \times W \rightarrow \mathscr{P}(W)
$$

and the following properties hold:

(A) $\gamma((x \circ y) \circ z)=\gamma(x \circ(y \circ z)), \gamma((x \curlywedge y) \curlywedge z)=\gamma(x \curlywedge(y \curlywedge z))$,

(E) $\gamma(E \circ x)=\gamma(\{x\})=\gamma(x \circ E)$,

(N) $x \circ y N z$ iff $x N(z / / y)$ iff $y N(x \backslash \backslash z)$

(D) $x \curlywedge y N z$ iff $x N(z \Leftarrow y)$ iff $y N(x \Rightarrow z)$

(I) $\gamma(x \curlywedge x)=\gamma(\{x\}), \gamma(x \curlywedge y) \subseteq \gamma(\{x\})$ and $\gamma(x \curlywedge y)=\gamma(y \curlywedge x)$.

The property $(\mathrm{N})$ is called the nuclear condition: it ensures that the closure operator $\gamma$ is a nucleus, i.e., satisfies $\gamma(X) \circ \gamma(Y) \subseteq \gamma(X \circ Y)$. The nucleus image of a residuated lattice is again a residuated lattice [GJKO07, Thm 3.34], which is important for the upcoming definition of Galois algebra. Likewise, property (D) is the distributive nuclear condition and ensures that $\gamma(X) \curlywedge \gamma(Y) \subseteq \gamma(X \curlywedge Y)$. Together with (I) it implies that $\gamma(X \curlywedge Y)=\gamma(X) \cap \gamma(Y)$, making the nucleus image a distributive lattice [GJ17, Lem. 2.1, 2.3].

The Galois algebra of $\mathfrak{W}$ is $\mathfrak{W}^{+}=\left(\gamma[\mathscr{P}(W)], \cap, \cup_{\gamma}, \circ_{\gamma}, 1, \backslash, /, \rightarrow\right)$ where

$$
\begin{aligned}
& -X \cup_{\gamma} Y=\gamma(X \cup Y), \\
& -X \circ \gamma=\gamma(X \circ Y), \\
& -X \backslash Y=\{z \in W: X \circ\{z\} \subseteq Y\},
\end{aligned}
$$


$-X / Y=\{z \in W:\{z\} \circ Y \subseteq X\}$ and

- $X \rightarrow Y=\{z \in W: X \curlywedge\{z\} \subseteq Y\}$.

To become familiar with the concept of a GBI-frame, it is a good exercise to prove the following important result.

Theorem 8.2 ([G.J17]) For any GBI-frame $\mathfrak{W}$ the Galois algebra $\mathfrak{W}^{+}$is a complete perfect GBI-algebra.

Conversely, given any GBI-algebra $\mathfrak{A},(A, A, \leq, \cdot,\{1\}, \backslash, /, \wedge, \rightarrow, \leftarrow)$ is a GBI-frame, where the operation $x \leftarrow y$ is defined as $y \rightarrow x$.

It is easy to see that when $W=W^{\prime}=A$ and $N=\leq$ then $\left(\gamma[\mathscr{P}(W)], \cap, \cup_{\gamma}\right)$ is the MacNeille completion of (the lattice reduct of) $\mathfrak{A}$, hence we immediately have the following result.

\section{Corollary 8.3 GBI is closed under MacNeille completions.}

We now outline a proof of algebraic cut elimination. We define a "free" GBI-frame $\mathfrak{W}_{F}$ with the property that any inequality $s \leq t$ that is satisfied in the Galois algebra $\mathfrak{W}_{F}^{+}$is provable from the rules of Table 8.1 without using the cut-rule. This definition illustrates that the concept of GBI-frame is flexible and provides frame-semantics for Gentzen proof systems.

Recall that $\mathfrak{F m}_{\mathrm{GBI}}$ is the absolutely free term algebra over the signature of GBI-algebras, and let $\mathfrak{W}$ be the homomorphic image such that $(W, \cdot, 1)$ is a free monoid and $(W, \wedge, \top, \perp)$ is a free commutative monoid with absorbing element $\perp$. Note that $W$ is itself an algebra with the signature of a GBI-algebra.

A $\cdot, \wedge$-unary linear polynomial on $W$ is a term with a single designated variable $x_{0}$ such that only the operations · and $\wedge$ appear on the branch from the root to $x_{0}$. Let $U$ be the set of all such terms, and define $W^{\prime}=U \times W$. We use the notation $u\left({ }_{-}\right)$for a polynomial $u \in U$, so e.g., $-\cdot y$ is the polynomial defined by $u\left(x_{0}\right)=x_{0} \cdot y$. The identity polynomial is denoted by $i d$.

Now define the relation $N \subseteq W \times W^{\prime}$ by

$$
x N(u, y) \quad \text { iff } \quad u(x) \leq y \text { is provable from Table } 8.1 \text {. }
$$

Then

$$
\begin{gathered}
x \cdot y N(u, z) \text { iff } u(x \circ y) \leq z \text { iff } x N(u(-\cdot y), z) \text { iff } y N\left(u\left(x \cdot \cdot_{-}\right), z\right), \\
x \wedge y N(u, z) \text { iff } u(x \wedge y) \leq z \text { iff } x N(u(-\wedge y), z) \text { iff } y N\left(u\left(x \wedge_{-}\right), z\right) .
\end{gathered}
$$

Hence we define

$$
\begin{aligned}
& x \circ y=\{x \cdot y\} \quad E=\{1\} \quad x \curlywedge y=\{x \wedge y\} \\
& (u, y) / / x=\{(u(-\cdot x), y)\} \quad x \backslash(u, y)=\left\{\left(u\left(x \cdot \cdot_{-}\right), y\right)\right\} \\
& (u, y) \Rightarrow x=\{(u(-\wedge x), y)\} \quad x \Leftarrow(u, y)=\left\{\left(u\left(x \wedge_{-}\right), y\right)\right\} \\
& \mathfrak{W}_{F}=\left(W, W^{\prime}, N, \circ, E, \|, / /, \curlywedge, \Rightarrow, \Leftarrow\right) \text {. }
\end{aligned}
$$

It is straightforward to show that $\mathfrak{W}_{F}$ satisfies (A), (E), (N), (D), (I), so it is a GBI-frame.

The following result is at the core of algebraic cut-elimination. For $y \in W^{\prime}$ we define $y^{\triangleleft}=\{x \in W: x N y\}$.

Lemma 8.4 Let $h: \mathfrak{W} \rightarrow \mathfrak{W}_{F}^{+}$be the unique homomorphism that extends the assignment $h\left(x_{i}\right)=\left(i d, x_{i}\right)^{\triangleleft}$. Then $t \in h(t) \subseteq(i d, t)^{\triangleleft}$ for all terms $t \in W$.

Proof This is proved by induction on the structure of $t$. For variables $x_{i}$ we have $x_{0} \in h\left(x_{0}\right)$ since $x_{0} \leq x_{0}$ is an axioms.

For the induction step, assume $s \in h(s) \subseteq(i d, s)^{\triangleleft}$ and $t \in h(t) \subseteq(i d, t)^{\triangleleft}$. We only check that

$$
s \rightarrow t \in h(s \rightarrow t) \subseteq(i d, s \rightarrow t)^{\triangleleft},
$$

since the remaining cases are similar.

Since $h$ is a homomorphism, we have

$$
\begin{aligned}
h(s \rightarrow t) & =h(s) \rightarrow h(t) \\
& =\{z \in W: h(s) \curlywedge z \subseteq h(t)\} .
\end{aligned}
$$


From $s \in h(s)$ we deduce that $z \in h(s \rightarrow t)$ implies

$$
\begin{aligned}
s \curlywedge z & =\{s \wedge z\} \\
& \subseteq h(t) \subseteq(i d, t)^{\triangleleft} .
\end{aligned}
$$

Therefore $s \wedge z \leq t$ is Gentzen provable, hence $z \leq s \rightarrow t$ is also provable by $\left[\rightarrow_{r}\right]$. We conclude that $z \in(i d, s \rightarrow t)^{\triangleleft}$ and thus

$$
h(s \rightarrow t) \subseteq(i d, s \rightarrow t)^{\triangleleft} .
$$

Next, let $(u, r) \in W^{\prime}$ and suppose $h(t) \subseteq(u, r)^{\triangleleft}$. Since $t \in h(t)$ it follows that $u(t) \leq r$ is Gentzen provable. Consider any $s^{\prime} \in h(s)$, whence $s^{\prime} \leq s$ is Gentzen provable. From $\left[\rightarrow_{l}\right]$ we see that

$$
u\left(s^{\prime} \wedge(s \rightarrow t)\right) \leq r
$$

is Gentzen provable. Therefore $s^{\prime} \wedge(s \rightarrow t) \in(u, r)^{\triangleleft}$.

Since every $\gamma$-closed set is an intersection of sets of the form $(u, r)^{\triangleleft}$, it follows that $s^{\prime} \wedge(s \rightarrow t) \in h(t)$ for all $s^{\prime} \in h(s)$. We conclude that

$$
h(s) \curlywedge(s \rightarrow t) \subseteq h(t),
$$

hence by definition of $\rightarrow$ in the Galois algebra it is the case that $s \rightarrow t \in h(s) \rightarrow h(t)=h(s \rightarrow t)$.

Theorem 8.5 For any s,t the following statements are equivalent.

(i) $\mathrm{GBI} \models s \leq t$,

(ii) $\mathfrak{W}_{F}^{+} \models s \leq t$,

(iii) $s \leq t$ has a cut-free proof using the rules in Table 8.1

Proof (i) implies (ii) since $\mathfrak{W}_{F}^{+}$is a GBI-algebra. Next, (ii) implies $h(s) \subseteq h(t)$, so $s \in h(s) \subseteq h(t) \subseteq(i d, t)^{\triangleleft}$ (Lemma 8.4). Hence $s N(i d, t)$ and therefore (iii) follows from the definition of $N$. Finally, (iii) implies (i) via Lemma 8.1

It takes more work to obtain a decision procedure for well-behaved subvarieties of $\mathrm{nGBI}$ (cf. $\S$ 7.1). The problem is that the rule $\left[\wedge_{i d e m}\right]$ could lead to an infinite branch during proof search. As for intuitionistic logic one can restrict to 3-reduced sequents, but one has to define a suitable measure for the length of a sequent to ensure that the sequents in the premise of a rule do not increase in length. For a detailed discussion on how to resolve these issues we refer to Galatos and Jipsen [GJ17] (see, however, Footnote77.

Remark 8.6 The paper in question also proves the finite model property for all subvarieties defined by identities in the language of $\{\cdot, \wedge, \vee, 1\}$, showing that they have a decidable equational theory. Moreover, if any of these varieties is integral (i.e., $x \vee 1=1$ ) then the finite embeddability property holds, hence such varieties have a decidable universal theory.

\section{9 (B)BI and Separation Logic}

We have not said much so far about the formalism that is largely responsible for the popularity of (B)BI in theoretical computer science: that is, about separation logic (SL). It is a form of Hoare logic for programs involving shared mutable data structures. We first recapitulate the basic ideas of general-purpose formalisms in $\S 9.1$, then we discuss specific issues addressed by SL and its cousins in $\S 9.2$ and finally get into the details of suitable Hoare-style reasoning in $\S 9.3$

While we believe this section is reasonably self-contained, the overview-aimed mostly at readers with limited background in program verification-must remain somewhat sketchy by nature. One can find more information in specialized overviews such as an early one by Reynolds [Rey02] or a more recent one by O'Hearn [OHe12]. 


$$
\begin{array}{ccc}
\{P\} \operatorname{SKIP}\{P\} & \{P[a / X]\} X:=a\{P\} & \frac{\{P\} C_{1}\{Q\} \quad\{Q\} C_{2}\{R\}}{\{P\} C_{1} ; C_{2}\{R\}} \\
\frac{\{P \wedge b\} C_{1}\{Q\}}{\{P\} \operatorname{IF} b \text { THEN } C_{1} \operatorname{ELSE} C_{2}\{Q\}} & \{P \wedge \neg b\} C_{2}\{Q\} & \{P \wedge b\} C\{P\} \\
\hline P \text { WHILE } b \text { DO } C \text { OD }\{P \wedge \neg b\}
\end{array}
$$

Table 9.1 Axioms and rules for commands of IMP

\subsection{Basic Ideas of Floyd-Hoare Logic(s)}

Floyd-Hoare logic [Flo67; Hoa69], most commonly abbreviated to Hoare logic, allows both writing specifications of programs and reasoning about their correctness using simple compositional rules. Its central notion is that of a partial correctness assertion (a.k.a. a Hoare triple) of the form $\{P\} C\{Q\}$, where precondition $P$ and postcondition $Q$ are logical predicates, written in some logical formalisms-it might be an extension of ordinary first-order logic or a variant or extension of (B)BI- and $C$ is a command, to be made specific below. A Hoare triple is valid if whenever $C$ is executed in a state satisfying $P$, it will terminate in a state satisfying $Q$.

Even when programs are not allowed to directly manipulate pointers, Hoare logic can be puzzling for a beginner. Textbook examples show it is easy to get the rules wrong for commands as simple assignment, especially when the assignment formula is allowed to involve the old value of a variable being assigned. Consider $\{\top\} X:=a\{X=a\}$. It might seem a valid triple scheme until one realizes that the expression $a$ can be, for example, $X+1$.

Let us begin with a typical toy programming language IMP used both in today's standard monographs [Pie+18; Win93] and classical references like Hoare's original paper [Hoa69]. It involves assignment, sequencing, conditionals and loops. IMP is not doing any (de)allocation, heap access, concurrency or operations on other shared mutable data structures; we will turn our attention to these below. An execution of an IMP program consists in manipulating (global) program "variables' 9 Par $=\left\{X_{0}, X_{1}, X_{2}, \ldots\right\}$ by assigning to them arithmetical expressions built using basic arithmetical operations (addition, optionally also multiplication and/or truncated subtraction) from $P$ Var and numerical constants. Conditionals and loops are guarded by boolean expressions $b_{0}, b_{1}, \ldots$ which are built using standard boolean connectives from atoms comparing arithmetical expressions for (in)equality. Finally, in the language of assertions (but not IMP itself!) we also allow class of another genuine, quantified assertion variables AVar $=\left\{v_{0}, v_{1}, v_{2}, \ldots\right\}$, for which one can substitute arithmetical expressions. This is enough to characterize all commands of IMP by axioms in Table 9.1 .

In addition, manipulation of the Hoare calculus requires rules that are, interestingly, often called structural [Kle99; ORY01; Rey02; COY07; OHe12]. Here, this word is taken in a somewhat different meaning than the one known to proof theorists. Namely, it denotes the rules which allow modifying pre- and post-conditions, as opposed to specifying complex program expressions in terms of their constituent subprograms along the lines of Table 9.1 Nevertheless, as we are going to see in $\S 9.3$, the central rule of separation logic connects this meaning of "structurality" with the one familiar to substructural logicians!

Perhaps the most well-known "structural" rule is consequence:

$$
\frac{\vDash P^{\prime} \rightarrow P \quad\{P\} C\{Q\} \quad \vDash Q \rightarrow Q^{\prime}}{\left\{P^{\prime}\right\} C\left\{Q^{\prime}\right\} .} \text { CONSEQ }
$$

Recall that the language of assertions includes quantified arithmetical statements. Hence, we use the semantic theoremhood $\vDash$ rather than the syntactic theoremhood $\vdash$ in the statement of this rule: there are obvious Gödelian limitations meaning there cannot be any complete yet recursively axiomatizable notion of proof (see however $\S 10.2$ below). Obviously, this entails that in practical applications one can only look for decision procedures for well-behaved fragments. Such limitative results also open up application areas for partially automated proof assistants as an alternative to fully automated tools.

${ }^{9}$ A logician may object whether the word variables is really appropriate here. Sometimes the term (storage) locations is used instead [Win93], but as the reader will recall, we already used this name in $\S 3.4$ for pointer labels and will continue to do so below. 
Remark 9.1 Semantics, either operational or denotational [Win93], can be given using the notion of store (stack) as introduced in $\S 3.4^{10}$ However, some proponents of Hoare-style formalisms point out that the above set of rules can be seen as semantics in its own right; hence the name axiomatic semantics (cf., e.g., [Win93, Ch. 6]). One can prove soundness theorems connecting it to more standard semantics. There is even a form of completeness available, although one has to tread carefully here due to the Gödelian limitations mentioned above: namely, these rules allow deriving suitable weakest preconditions and consequently all valid IMP-triples in the presence of an oracle for elementary arithmetic. This is called relative completeness [Coo78] (see also [Cla85], [Win93, Ch. 7]). We will continue the discussion of semantical aspects of correctness assertions in Example 9.2 and Remark 9.3 below.

The popularity of Hoare logics, however, does not stem so much from theoretical results like relative completeness (available only in a restricted context anyway [Cla85]), but from its applications to program specification and verification. For example, they allow (semi-)automated verification of programs annotated/decorated with assertions via extraction of verification conditions [Win93, § 7.4], [Pie+18]. There are programming languages with specification constructs built-in, like Eiffel [Mey97] or Dafny [Lei10], but for scalable analysis of industrial-size code in a general-purpose language one uses analysis platforms like Frama-C [Cuo+12], allowing annotations written in an external specification language (e.g., ACSL).

So much for bird's eye view of general-purpose (Floyd-)Hoare logic(s). Now where and how does the connection with (B)BI enter the picture?

\subsection{Heap(let)s, Allocation and Separation}

As we have already indicated, specification and automated verification become particularly problematic in the presence of shared mutable data structures. O'Hearn, Reynolds and Yang [ORY01] summarized this as follows:

The main difficulty is not one of finding an in-principle adequate axiomatization of pointer operations; rather there is a mismatch between simple intuitions about the way that pointer operations work and the complexity of their axiomatic treatments. For example, pointer assignment is operationally simple, but when there is aliasing, arising from several pointers referring to a given cell, then an alteration to that cell may affect the values of many syntactically unrelated expressions.

The idea that substructural connectives can help axiomatic approaches to the assertion language can be traced back to Burstall [Bur72]. Much later, Reynolds [Rey00; Rey02] and Ishtiaq and O'Hearn [IO01] turned the idea into a working, well-defined Hoare-style language. These papers also provide more references to earlier attempts at a suitable verification logic (cf. $\S 12.1$ ).

To understand the advantages of assertions expressed in (an extension of) (B)BI, let us continue the above quote from O'Hearn et al. [ORY01]:

We suggest that the source of this mismatch is the global view of state taken in most formalisms for reasoning about pointers. ... To understand how a program works, it should be possible for reasoning and specification to be confined to the cells that the program actually accesses. The value of any other cell will automatically remain unchanged.

Thus, substructural connectives are used to express and combine assertions about disjoint portions of heap: an assertion talks about...

... a heaplet rather than the global heap, and a spec $\{P\} C\{Q\}$ says that if $C$ is given a heaplet satisfying $P$ then it will never try to access heap outside of $P$ (other than cells allocated during execution) and it will deliver a heaplet satisfying $Q$ if it terminates. [BCO06]

\footnotetext{
${ }^{10}$ Note that the assertion for WHILE is valid only when read as a partial one. That is, $\{P\} C\{Q\}$ is read as if $C$ is started in a store satisfying $P$ and terminates, then any store it terminates in satisfies $Q$. Under this reading, for example, $\{\top\} C\{\perp\}$ simply specifies that $C$ never terminates, regardless of the original values of program variables. An alternative reading, usually denoted as $[P] C[Q]$, is the total one: if $C$ is started in a store satisfying $P$, then it terminates and any store it terminates in satisfies $Q$
} 
It is easy to guess now that assertions about disjoint heaplets are combined using the fusion $*$ of commutative (B)BI, which in this community has alternative names like the spatial conjunction, the separating conjunction or the independent conjunction. Its residual $-*$ is commonly referred to as the magic wand or separating implication: $P-* Q$ means whenever the present heaplet is extended with a disjoint heaplet satisfying $P$, the resulting heaplet satisfies $Q$. The use of $-*$ in Hoare triples of separation logic (SL) seems to have been proposed first by Ishtiaq and O'Hearn [IO01]. Just like its additive counterpart, $-*$ is particularly useful when specifying and deriving weakest preconditions 11

For a concrete example of a suitable programming language equipped with a Hoare logic, let us take our inspiration from Reynolds [Rey02]. And for a semantic intuition, let us return to models of (B)BI discussed in $\S 3.4$, especially the stack-and-heap model [BK14, § 2.2] introduced at the end of that subsection. As we pointed out therein, the name store also used by, e.g., Demri and Deters [DD15a] would be perhaps more adequate, hence we speak about the store-and-heap model instead. In order to allow full pointer arithmetic, let us identify locations, record values and store values, i.e., (in the notation of $\S 3.4$ ) take $L=R V=V a l=\mathbb{N}$. The advantage of operational semantics based on such a model (i.e., on a set-theoretic product of the collection of stores and the collection of heaps) is that it allows extending IMP with dynamic commands in a fully orthogonal way. Of course, as we incorporate IMP with its assertion language, Gödel's Incompleteness Theorem still applies; in $\S 10.2$ below, we discuss restrictions allowing more positive results.

We take thus IMP from $\S 9.1$ and add primitives for

allocation $X:=\operatorname{CONS}\left(a_{0}, \ldots, a_{n-1}\right)$, lookup $X:=[a]$, mutation $[a]:=a^{\prime}$, and deallocation DISPOSE $a$.

We also take the entire assertion language introduced in $\S 9.1$ and allow the use of $*$ and $-*$ to form new assertions. Furthermore, we extend the language of assertions with new atomic constructs:

- a constant emp true at any pair $(s, \emptyset)$ where $\emptyset$ is the empty heap(let) and

- a family of pointer atoms $a_{1} \mapsto a_{2}$ which hold at those pairs $(s, h)$ where $h$ is a singleton heap(let), i.e., defined on exactly one location, which happens to be $\hat{s}\left(a_{1}\right)$ ( $\hat{s}$ denotes the inductive extension of $s$ to arbitrary arithmetical expressions) and $h\left(\hat{s}\left(a_{1}\right)\right)=\hat{s}\left(a_{2}\right)$.

The presence of $*$ in the language means we can describe any concrete finite heap using expressions of the form $\left(a_{1} \mapsto e_{1}\right) * \cdots *\left(a_{n} \mapsto e_{n}\right)$. Given $\bar{a}:=a_{0}, \ldots, a_{n-1}$, let us also introduce an abbreviation for $e$ pointing to the head of a dynamic list storing numbers denoted by $\bar{a}$ :

$$
e \mapsto_{\ell} \bar{a}:=\left(e \mapsto a_{0}\right) *\left(e+1 \mapsto a_{1}\right) * \cdots *\left(e+(n-1) \mapsto a_{n-1}\right)
$$

Reynolds [Rey02] or Ishtiaq and O'Hearn [IO01] point out that the validity of $\{P\} C\{Q\}$ (whether as partial or total correctness assertions, cf. Rem. 9.1 and especially Footnote 10) should entail not only that whenever $P$ holds at a pre-execution state $(s, h)$, then $Q$ would hold after the execution of $C$, but also that $C$ executed at $(s, h)$ is safe, i.e., does not lead to a memory fault. That is, it should never try to mutate, lookup or dispose of a cell which has not been previously allocated 12

Under this reading, we can salvage all of the rules for IMP discussed in $\S 9.1$ though some of the clauses (especially the one for WHILE) require more work due to the safeness requirement. Moreover, we can finally give the axiomatic semantics of new, dynamic constructs in Table 9.2. Note here how we use the auxiliary, quantifiable variables of the metalanguage to keep original values of $X$ in the allocation and lookup clauses. Recall again from $\S 9.1$ that in distinguishing program "variables" $P$ Var from variables of the metalanguage AVar we follow standard references like the Winskel book [Win93] rather than, e.g., the presentation of Reynolds [Rey02]. A similar approach to ours is also taken by Demri and Deters [DD15a].

\footnotetext{
${ }^{11}$ On the other hand, the semantic clause of $-*$ quantifies over the collection of all possible disjoint extensions satisfying $Q$, which can be infinite, and is problematic from a model checking point of view. For this reason, there is a line of research dealing with adjunct elimination for separation logic and related formalisms [Loz04; DGG04; CGZ07; CDG10].

${ }^{12}$ When specifying this property in a proof assistant, one can do it in a (co)inductive fashion. In a metatheory allowing excluded middle at least for assertions, one can work with two inductive properties fault and no_fault and show (using excluded middle) that they are complementary, i.e., that for any $(s, h)$ and $C$ exactly one of the two holds. Alternatively, one can stay within constructive metatheory by making no_fault coinductive. See [Pau16] for an example of a student-oriented formalization in a proof assistant discussing these issues.
} 


$$
\begin{gathered}
\{(X=v) \wedge e m p\} X:=\operatorname{CONS}(\bar{a})\left\{X \mapsto_{\ell}(\bar{a}[v / X])\right\}, \\
\left\{(X=v) \wedge\left(a \mapsto v^{\prime}\right)\right\} X:=[a]\left\{\left(X=v^{\prime}\right) \wedge a[v / X] \mapsto v^{\prime}\right\}, \\
\{\exists v \cdot a \mapsto v\}[a]:=a^{\prime}\left\{a \mapsto a^{\prime}\right\}, \\
\{\exists v \cdot a \mapsto v\} \text { DISPOSE } a\{e m p\} .
\end{gathered}
$$

Table 9.2 Small or local axioms for dynamic commands.

\subsection{Local Axioms, Global Specifications and The Frame Rule}

The axioms in Table 9.2 are local or small (cf., e.g., [OHe12]). A specification for $C$ is local if only involves variables used by $C$ and what O'Hearn called the footprint of $C$ : parts of the heap used during its execution. While it is easy to see why the modular approach postulated at the beginning of this section requires such small axioms, it also calls for suitable structural rules in the sense already mentioned in $\S 9.1$. We need a rule which allows deriving triples of the form $\{P * R\} C\{Q * R\}$ from triples of the form $\{P\} C\{Q\}$. In other words, the central structural rule of separation logic must be very much a structural rule in ordinary proof-theoretic sense: an introduction rule for $*$.

Nevertheless, without suitable restrictions, such an inference can be unsound if $C$ involves an allocation, lookup or assignment to some $X$ (i.e., with $X$ on the left side of “:=”) appearing in $R$.

Example 9.2 Consider, for example, $C$ being $X:=\operatorname{CONS}(2), P$ and $Q$ being the constantly true assertion $\top$ and $R$ being the assertion $X=2$. We do have that $\vDash\{\top\} X:=\operatorname{CONS}(2)\{\top\}, 13$ but

$$
\not \models\{\top *(X=2)\} X:=\operatorname{CONS}(2)\{\top *(X=2)\} .
$$

To understand (9.1), recall that the execution of $X:=\operatorname{CONS}(\bar{a})$ in $(s, h)$ can transition to any $\left(s^{\prime}, h^{\prime}\right)$, where $h^{\prime}$ is obtained by extending $h$ with a contiguous interval of fresh heap addresses pointing at the (values denoted at $s$ by) subsequent elements of $\bar{a}$, and $s^{\prime}$ is just $s$ modified at $X$ to store the newly allocated address of the first element of the list. In the case of a singleton list $\bar{a}=[2]$, we can start with $h$ being empty and $h^{\prime}$ consisting, e.g., of a single pair $(1,2)$, meaning that $s^{\prime}(X)=1$, even if $s(X)=2$. We thus obtain an example of a (non-faulting) execution starting in a state satisfying the precondition and resulting in a state where the postcondition fails-a counterexample to the validity of the triple.

Define appear $(R)$ as the set of program variables syntactically appearing 14 in the assertion $R$ and the predicate modifies as shown in Table 9.3 . The frame rul 15 proposed by O'Hearn [ORY01; [O01] is hence

$$
\frac{\{P\} C\{Q\} \quad \text { modifies }(C) \cap \text { appears }(R)=\emptyset}{\{P * R\} C\{Q * R\}} \text { Frame }
$$

Remark 9.3 For a logician and perhaps even more so for an algebraist, the presence of side conditions such as modifies $(C) \cap$ appears $(R)=\emptyset$ is certainly disappointing. This sentiment is shared by theoretical computer scientists:

Hoare logic is bedevilled by complex but coarse side conditions on the use of variables. [PBC06]

\footnotetext{
${ }^{13}$ In our ideal mathematical world, where heaps can be arbitrarily large as long as they are finite, allocation never leads to a memory fault (unlike lookup, mutation and deallocation).

${ }^{14}$ Of course, only free occurrences matters. But in our language there are no binders for elements of PVar (unlike AVar).

15 The term frame problem was originally proposed in a classical 1969 paper [MH69] on problems of knowledge representation in artificial intelligence. The realization that such problems arise also in formal specifications using Floyd-Hoare logics predates the development of separation logic. An example, focusing on the issues of object-oriented specifications with inheritance, is provided by a 1995 paper by Borgida et al. [BMR95].
} 


$$
\begin{aligned}
\text { modifies }(X:=a) & =\text { modifies }(X:=[a]) \\
& =\operatorname{modifies}(X:=\operatorname{CONS}(\bar{a}))=\{X\}, \\
\text { modifies }(\operatorname{SKIP}) & =\operatorname{modifies}(\operatorname{DISPOSE} a) \\
& =\operatorname{modifies}\left([a]:=a^{\prime}\right)=\emptyset, \\
\text { modifes }\left(C_{1} ; C_{2}\right) & =\operatorname{modifies}\left(\operatorname{IF} b \operatorname{THEN} C_{1} \operatorname{ELSE} C_{2}\right) \\
& =\text { modifies }\left(C_{1}\right) \cup \operatorname{modifies}\left(C_{2}\right), \\
\text { modifies }(\text { WHILE } b \text { DO } C \text { OD }) & =\text { modifies }(C) .
\end{aligned}
$$

Table 9.3 Predicate modifies.

Some readers may be puzzled by the fact that in some references (e.g., [COY07]) the frame rule is stated without side conditions nonetheless. Calcagno et al. [COY07, $\S 1$ ] claim that such conditions can be avoided thanks to the absence of "the traditional Hoare logic punning of program variables as logical variables", crediting [BCY06; $\mathrm{PBC} 06]$ with the idea.

The quote might be somewhat confusing, depending on what punning of program variables as logical variables is taken to mean. Recall once again that in our setting we do distinguish between program variables (storage locations, cf. Footnote 9) PVar $=\left\{X_{1}, X_{2}, X_{3} \ldots\right\}$ and quantified assertion variables $A \operatorname{Var}=\left\{v_{1}, v_{2}, v_{3} \ldots\right\}$. More informative descriptions of the problem are

[Program] variables ought to be resource, treated formally by the logic and not mumbled over in side conditions. [BCY06]

and, still more precisely,

Hoare logic does not allow us to describe the ownership of [program] variables ... Separation logic divides the store into stack - the variables used by a program - and heap-dynamically allocated records_-but does not give any formal treatment of the stack. [PBC06]

Returning with this insight to Example 9.2, we can see that the use of $*$ in pre- and post-conditions is entirely irrelevant in 9.1): $X$ lives in the store ("stack"), not on the heap, and any (in)equality statement about its value at a given $(s, h)$ will be also valid at $\left(s, h^{\prime}\right)$, for any other $h^{\prime}$. In other words, equality judgements, including those involving members of PVar, "spread beyond the separating conjunction"; they are heapindependent. The syntactic apparatus of SL is indeed not tailored to improve control of the store. Hence, avoiding problematic side conditions not only departs "from the theoretical tradition in program logic" [COY07], but necessitates restricting/complicating the assertion language and the programming language in question. The setup of Calcagno et al. [COY07], for example, does not cover alteration of programming variables, whereas that of Parkinson et al. [PBC06] not only relies on explicit "ownership" predicates, but also on side conditions more familiar in algebra and logic, i.e., standard freshness assumptions. Still, such approaches are particularly relevant in applications of separation logic focusing on concurrency rather than pointer reasoning (cf. $\S 12.3$ ).

With the frame rule at our disposal, we can derive global specifications from local ones-and in the process use basic axioms and rules of (B)BI. We are providing these inferences explicitly in Table 9.4 below; to save some space, we abbreviate " $\exists v . a \mapsto v$ ” as " $a \mapsto$ _." Such derivations are outlined, e.g., by Reynolds [Rey02], Yang [Yan01] or O'Hearn [OHe12]. Let us discuss their most salient points.

Deallocation is rather straightforward: $\left\{\left(a \mapsto_{-}\right) * R\right\}$ DISPOSE $a\{R\}$. Its derivation in Table 9.4 uses the fact that emp is the monoidal unit. One arrives almost instantly at the form suitable for backward reasoning [IO01], i.e., allowing backwards program annotations, starting from an arbitrary postcondition $R$. Such reasoning is at the heart of many applications of Hoare-style formalisms, in particular derivations of weakest preconditions, which in turn are essential for relative completeness results (cf. Remark 9.1). A discussion of such results in the context of separation logic was provided in an early stage of its development by Yang [Yan01]. 

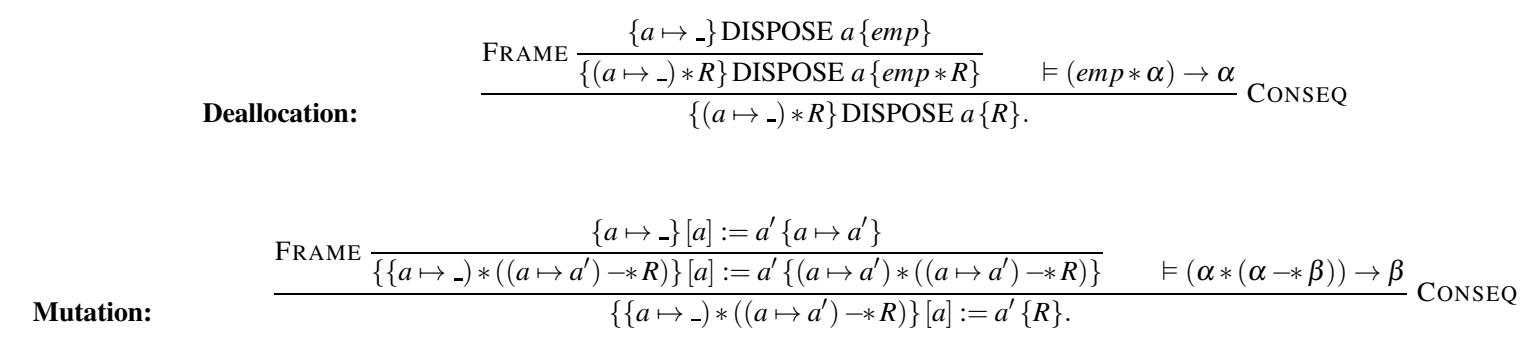
FrAmE $\frac{\left\{(X=v) \wedge\left(a \mapsto v^{\prime}\right)\right\} X:=[a]\left\{\left(X=v^{\prime}\right) \wedge a[v / X] \mapsto v^{\prime}\right\}}{\underline{\left\{\left((X=v) \wedge\left(a \mapsto v^{\prime}\right)\right) * \alpha\left[v^{\prime} / X\right]\right\} X:=[a]\left\{\left(\left(X=v^{\prime}\right) \wedge a[v / X] \mapsto v^{\prime}\right) * \alpha\left[v^{\prime} / X\right]\right\}} \vDash\left(\left(X=v^{\prime}\right) \wedge \beta\right) * \gamma\left[v^{\prime} / X\right] \rightarrow \beta * \gamma}$ CONSEQ $\left\{\left((X=v) \wedge\left(a \mapsto v^{\prime}\right)\right) * \alpha\left[v^{\prime} / X\right]\right\} X:=[a]\left\{\left(a[v / X] \mapsto v^{\prime}\right) * \alpha\right\}$

\begin{tabular}{cc}
\hline$\left\{\left((X=v) \wedge\left(a \mapsto v^{\prime}\right)\right) * \alpha\left[v^{\prime} / X\right]\right\} X:=[a]\left\{\left(a[v / X] \mapsto v^{\prime}\right) * \alpha\right\}$ & CONSEQ \\
\hline$\left\{\left((X=v) \wedge\left(a \mapsto v^{\prime}\right)\right) *\left(\left(a[v / X] \mapsto v^{\prime}\right)-* R\left[v^{\prime} / X\right]\right)\right\} X:=[a]\left\{\left(a[v / X] \mapsto v^{\prime}\right) *\left(\left(a[v / X] \mapsto v^{\prime}\right)-* R\right)\right\}$ & SUBST \\
$\vDash(X=v) \wedge(\beta * \gamma) \rightarrow((X=v) \wedge \beta) * \gamma[v / X] \quad \vDash(\alpha *(\alpha-* \beta)) \rightarrow \beta$ & CONSEQ \\
\hline$\left\{(X=v) \wedge\left(\left(a \mapsto v^{\prime}\right) *\left(\left(a \mapsto v^{\prime}\right)-* R\left[v^{\prime} / X\right]\right)\right)\right\} X:=[a]\{R\}$ & VAREL
\end{tabular}
Lookup: $\left\{\left(a \mapsto v^{\prime}\right) *\left(\left(a \mapsto v^{\prime}\right)-* R\left[v^{\prime} / X\right]\right)\right\} X:=[a]\{R\}$

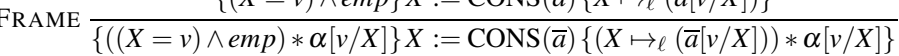

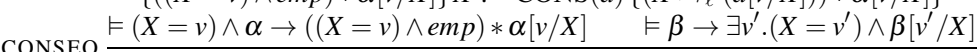

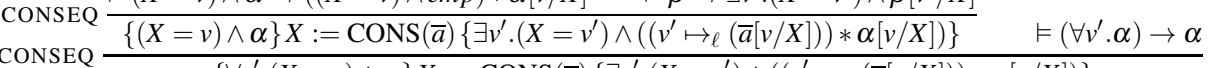

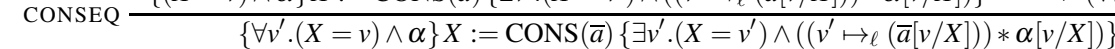
$\overline{\left\{\forall v^{\prime} .(X=v) \wedge\left(\left(v^{\prime} \mapsto_{\ell}(\bar{a}[v / X])\right)-* R\left[v^{\prime} / X\right]\right)\right\} X:=\operatorname{CONS}(\bar{a})\left\{\exists v^{\prime} .\left(X=v^{\prime}\right) \wedge\left(\left(v^{\prime} \mapsto_{\ell}(\bar{a}[v / X])\right) *\left(\left(v^{\prime} \mapsto_{\ell}(\bar{a}[v / X])\right)-* R\left[v^{\prime} / X\right]\right)\right)\right\}}$ SUBST $\vDash(X=v) \wedge \alpha[v / X] \rightarrow \alpha \quad \vDash(\alpha *(\alpha-* \beta)) \rightarrow \beta$

$\left.\left\{\forall v^{\prime} .\left(v^{\prime} \mapsto \varrho \bar{a}\right)-* R\left[v^{\prime} / X\right]\right)\right\} X:=\operatorname{CONS}(\bar{a})\left\{\exists v^{\prime} .\left(X=v^{\prime}\right) \wedge R\left[v^{\prime} / X\right]\right\}$ $\left.F\left(\exists v^{\prime} .\left(X=v^{\prime}\right) \wedge \alpha\left|v^{\prime}\right| X\right]\right) \rightarrow \alpha$

Allocation:

$\left.\left\{\forall v^{\prime} .\left(v^{\prime} \mapsto_{\ell} \bar{a}\right)-* R\left[v^{\prime} / X\right]\right)\right\} X:=\operatorname{CONS}(\bar{a})\{R\}$

Table 9.4 Derivations of global backwards specifications for local (small) axioms. 
The global backward specification for mutation:

$$
\left\{\left(a \mapsto_{-}\right) *\left(\left(a \mapsto a^{\prime}\right)-* R\right)\right\}[a]:=a^{\prime}\{R\},
$$

while still very simple to derive, is the first one where we need residuation:

$$
\vDash(P *(P-* Q)) \rightarrow Q
$$

(note that in Table 9.4 we often use Greek letters as metavariables ranging over assertions, if we want to instantiate them in the next step; we sometimes denotes this act of substitution as SUBST).

The global backward specification for lookup:

$$
\left\{\left(a \mapsto v^{\prime}\right) *\left(\left(a \mapsto v^{\prime}\right)-* R\left[v^{\prime} / X\right]\right)\right\} X:=[a]\{R\}
$$

requires a bit more effort. Apart from using again (9.2), instances of the consequence rule used in the derivation also use laws governing interactions of PVar, AVar, lattice and substructural connectives and heap-independent assertions, such as the equivalence:

$$
\vDash(X=v) \wedge(P * Q) \leftrightarrow((X=v) \wedge P) * Q[v / X] .
$$

Moreover, we also need an application of the (derivable or admissible) rule

$$
\frac{\{(X=v) \wedge P\} C\{R\} \quad v \text { fresh for } P, R}{\{P\} C\{R\}} \text { VAREL }
$$

In references like Reynolds [Rey02] or Yang [Yan01], there are special rules like auxiliary variable renaming and auxiliary variable elimination which can be used to derive such rules. In $\S 10.1$, we present another proof system where this rule is indeed derivable rather than primitive.

Similarly, obtaining the global backward axiom for allocation

$$
\left.\left\{\forall v^{\prime} \cdot\left(v^{\prime} \mapsto_{\ell} \bar{a}\right)-* R\left[v^{\prime} / X\right]\right)\right\} X:=\operatorname{CONS}(\bar{a})\{R\}
$$

requires using the frame and consequence rules jointly with BI laws and basic quantification laws, in particular $\vDash\left(\exists v^{\prime} .\left(X=v^{\prime}\right) \wedge R\left[v^{\prime} / X\right]\right) \rightarrow R$.

\section{Proof Theory and Decidability for Fragments of SL}

We have argued that proof theory of separation logic can be seen as an extension of proof theory of BI and substructural logics. It would be misleading, however, to give the impression that the only potential rôle of proof theory lies in deriving general axioms like those discussed above. If that were so, the reader may ask, why not simply begin with postulating the axioms in a suitable "global backwards" form? Furthermore, such a critical reader may be perplexed by questions of decidability, both in the light of the discussion in $\S 9.1$ and the one in $\S 7$. In this section, we are going to address both issues.

\subsection{Sketch of a Proof System for SL}

As we stated in $\S 9.1$ the industrial importance of Floyd-Hoare logics indeed does not quite stem from relative completeness results via calculation of schemes of weakest preconditions. While such results are an important theoretical characterization, the real practical interest lies in deriving and verifying annotations and specifications of concrete programs.

Let us then take stock recapitulating which axioms and rules were exactly used in Table 9.4. The resulting proof system can derive not only these "global backwards specifications", but-as the reader can verify-meaningful pieces of annotated code, similar to those used as examples, e.g., in [Rey02]. We propose that judgements $\vdash\{P\} C\{Q\}$ are deduced using the following axioms and rules:

- axioms in Tables 9.1 and 9.2. 
- the FRAME rule;

- rules

$$
\frac{\vdash\{P\} C\{R\} \quad v \text { fresh for } R}{\vdash\{\exists v \cdot P\} C\{R\}} \text { VAREL } \exists
$$

and

$$
\frac{\vdash\{P\} C\{R\} \quad v \text { fresh for } P}{\vdash\{P\} C\{\forall \cdot R\}} \text { VAREL } \forall ;
$$

- a deductive version of the consequence rule

$$
\frac{P^{\prime} \Rightarrow P \quad \vdash\{P\} C\{Q\} \quad Q \Rightarrow Q^{\prime}}{\vdash\left\{P^{\prime}\right\} C\left\{Q^{\prime}\right\},} \text { CONDED }
$$

where $P^{\prime} \Rightarrow P$ and $Q \Rightarrow Q^{\prime}$ are derived using:

- axioms and rules of any proof system which is equipollent with (can derive all theorems of) the Hilbert-style system for BI presented in $\S 2$ To fix attention, let us take the system presented in $\S 8$

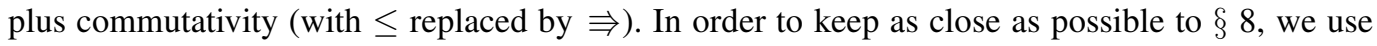
here the notation $\alpha(\varphi)$ where $\alpha$ denotes a bunch from $\S 8$ adjusted to the present syntax;

- the basic theory of equality (cf. [TS96, $\S 4.7]$ ), i.e.,

$$
\frac{\alpha(a=a) \Rightarrow \varphi}{\alpha(\top) \Rightarrow \varphi}
$$

and

$$
\frac{\alpha\left(a_{1}=a_{2}, \psi\left[a_{1} / v\right], \varphi\left[a_{2} / v\right]\right) \Rightarrow \chi}{\alpha\left(a_{1}=a_{2}, a_{1}=a_{2} \wedge \psi\left[a_{1} / v\right], a_{1}=a_{2} \wedge \varphi\left[a_{2} / v\right]\right) \Rightarrow \chi}
$$

where $\alpha(x, y, z)$ is the ternary counterpart of the notion of a bunch $h^{16}$ from $\S 8$ i.e., a scheme of an assertion formula in which each of the schematic variables $x, y$ and $z$ occurs only once, and on the term-tree branches where $x, y$ and $z$ occur, only the symbols $*$ and $\wedge$ are allowed;

- basic quantification rules (cf. [TS96, § 3.5]), i.e.,

- $\frac{\alpha(\varphi[a / v] \wedge \forall v \cdot \varphi) \Rightarrow \psi}{\alpha(\forall v \cdot \varphi) \Rightarrow \psi} \quad$ and $\quad \frac{\varphi \Rightarrow \psi[a / v]}{\varphi \Rightarrow \exists v \cdot \psi}$

- $\frac{\varphi \Rightarrow \psi}{\varphi \Rightarrow \forall v \cdot \psi}$ and $\frac{\alpha(\psi) \Rightarrow \varphi}{\alpha(\exists v \cdot \psi) \Rightarrow \varphi}$ whenever $v$ is fresh for $\varphi$ and $\alpha$;

- while we have not needed such axioms and rules in the derivations presented so far, any system used for reasoning about simple programs is likely to need additional principles governing pointer axioms - at the very least, some variant of a rule encoding disjointness of heaps:

$$
\frac{\alpha\left(e \mapsto a_{1} * e \mapsto a_{2}\right) \Rightarrow \varphi}{\alpha(\perp) \Rightarrow \varphi}
$$

and a rule encoding functionality of pointers:

$$
\frac{\alpha\left(e \mapsto a_{1} \wedge e \mapsto a_{2}\right) \Rightarrow \varphi}{\alpha\left(a_{1}=a_{2} \wedge e \mapsto a_{1}\right) \Rightarrow \varphi}
$$

- finally, as a parameter in the definition of our proof system, we allow the user to choose a bunchedsequent-style formulation of a fragment of arithmetic with good proof-theoretic properties, like Skolem's primitive recursive arithmetic (PRA) [TS96, § 4.5.2] or a chosen fragment of Presburger's arithmetic. Note again that it makes perfect sense to work with fragments which do not allow unrestricted pointer arithmetic, thus removing the need for incorporating arithmetic in our proof

\footnotetext{
${ }^{16}$ Note that this rule allows to spread equality statements across the bunch. In the store-and-heap semantics, such atoms are heap-independent: they only depend on the store.
} 
system. All we needed for inference rules and derivations so far was the ability to encode $e \mapsto_{\ell} \bar{a}$ and this we could do having only the syntax and axioms of the successor function; as discussed in $\S 10.2$ below one can go still further than that, take each $e \mapsto_{\ell} \bar{a}$ to be an atom in its own right and even restrict the length of $\bar{a}$ in such an expression. Even with no arithmetic present, when one is taking $\mathrm{BI}$ rather than BBI as the propositional base, it is natural to enrich the system so that one can derive the law of excluded middle for equality statements.

Note here that there are candidates for rules which can be admissible, but not necessarily derivable. O'Hearn et al. [OYR09, § 3.2.1] give as an example what they call the the conjunction rule:

$$
\frac{\left\{P_{1}\right\} C\left\{R_{1}\right\} \quad\left\{P_{2}\right\} C\left\{R_{2}\right\}}{\left\{P_{1} \wedge P_{2}\right\} C\left\{R_{1} \wedge R_{2}\right\}} .
$$

Let us observe that in the presence of the consequence rule this rule is clearly suboptimally formulated, just like auxiliary variable renaming or auxiliary variable elimination [Rey02; Yan01] derivable in our system. If one wants this rule to be derivable, it is enough to add

$$
\frac{\{P\} C\left\{R_{1}\right\} \quad\{P\} C\left\{R_{2}\right\}}{\{P\} C\left\{R_{1} \wedge R_{2}\right\}} .
$$

This indicates a more general pattern of rules for assertions mimicking sequent-style rules, where the command itself plays a rôle similar to that of a turnstile (inequality) sign. VAREL $\exists$ and VAREL $\forall$ above follow the same pattern. Yet another one, also sound over the intended semantics, would be

$$
\frac{\left\{P_{1}\right\} C\{R\} \quad\left\{P_{2}\right\} C\{R\}}{\left\{P_{1} \vee P_{2}\right\} C\{R\}} .
$$

It is not immediately obvious whether all such rules are admissible in the proposed system. Note that the restrictions necessary to ensure soundness of the frame rule indicate that only translations of the rules governing additive connectives are worth considering in this context.

\subsection{Decidability Revisited}

We have already noted in $\S 9.1$ that whenever the assertion language contains arithmetic (or anything sufficiently rich to encode it), Gödel's Incompleteness Theorem obviously implies that the set of valid of assertions cannot be even recursively enumerable. As we have discussed above, one sensible strategy is to focus on incomplete proof systems or decision procedures - and there is no shortage of useful heuristics. But we have also indicated that especially when reasoning about typical operations on shared mutable data structures-like linked list reversal or copying/deletion of a tree-one hardly ever needs full pointer arithmetic. Consequently, one can deal with assertion languages which are not automatically covered by Gödel's result. To improve the situation even more, one can further restrict the assertion language, e.g., by limiting the number of quantified variables. The limit case is reached when there are no quantified variables left: all the expressions of the assertion language constructed without the use of multiplicative (a.k.a. spatial, separating or simply substructural) connectives can be used verbatim as guards of WHILE or IF expressions of the programming language.

It would seem that such a propositional setup is precisely the one we have considered in $\S 7$ hence undecidability limits discussed therein still apply. But an astute reader may have already recalled Remark 7.4 the absence of uninterpreted algebraic variables standing for arbitrary propositions limits direct applicability of such purely algebraic results. And indeed, expressions of the assertion language of SL are built from concrete atoms of the form $a_{1}=a_{2}$, emp or $e \mapsto_{\ell} \bar{a}$ (note again that if we do not assume that the assertion language can directly encode at least the successor function, we need to allow a more general form of pointer atoms). How do we know that in at least some of the simpler boolean allocation/heap models of $\S \S 3.3$ and 3.4 such a restriction does not rule out valuations crucial for establishing undecidability of the set of BBI formulas valid in that model? 
As it turns out, this is precisely what happens. Calcagno, Yang and O'Hearn [CYO01, $\S \S 4-5]$ show that the quantifier-free BBI language obtained by restricting the pointer atoms to the binary form $e \mapsto \mapsto_{\ell} a_{1}, a_{2}$ (and with no function symbols) interpreted over store-and-heap models where $R V=L \cup\{$ nil $\}$ and heaps are finite partial functions from $L$ to $R V \times R V$ is PSPACE-complete, with further restrictions allowing even better complexity. By contrast, the set of all valid assertions in the quantified version of this language is not even recursively enumerable [CYO01, Th. 1]. A detailed discussion of this phenomenon is provided by Brotherston and Kanovich [BK14, $\S 10]$, who show how restriction to finite valuations in heap models can restore decidability for the propositional language 17 Demri and Deters [DD15a, $\S 4.3 .2$ ] note that an analogous PSPACE-completeness result holds with pointer atoms of the form $e \mapsto_{\ell} a_{1}, \ldots, a_{k}$ for arbitrary but fixed finite $k$. Furthermore, Demri et al. [DGLM14] show that when only pointer atoms of the form $e \mapsto a_{1}$ are allowed, the PSPACE upper bound survives in the presence of just one quantified variable (however, this result cannot be combined with atoms of the form $e \mapsto_{\ell} a_{1}, a_{2}$ [DD15b]). For more positive and negative decidability results for various fragments of the assertion language, the reader is referred to the overview of Demri and Deters [DD15a].

\section{Bi-Abduction: The Main Issue of SL Proof Theory}

The story of algorithmic questions dealt with by Separation Logic would be incomplete if we finished it here. We are now in a position to briefly discuss perhaps the most important proof-theoretic tasks for SL practitioners, which may be somewhat novel for more traditionally oriented algebraists and logicians. In the words of Peter O'Hearn (p.c.),

[t]he one thing I wish we could get across to substructural logicians is the importance of inference questions beyond validity. Chief among these are frame inference and abduction. [OHe]

To be sure, abductive inference is not exactly an unknown concept in philosophy and logic, its study dating back to Charles S. Peirce, with the term "used in two related but different senses" [Dou17] regarding the use of explanatory reasoning in either generating or justifying hypotheses. As stressed by, e.g., the corresponding entry in the Stanford Encyclopedia of Philosophy [Dou17], contemporary philosophers of science tend to employ it in the latter meaning (context of justification or inference to the best explanation), whereas Peirce himself 18 put it in the context of discovery:

Abduction is the process of forming explanatory hypotheses. It is the only logical operation which introduces any new idea (...) Abduction must cover all the operations by which theories and conceptions are engendered. [Pei58, CP 5.172,5.590]

It seems safe to say that the meaning of the term as used in computer science and artificial intelligence [Pau93; DK00; CDOY11] either combines the context of discovery with that of justification or even focuses specifically on the former one, thus being closer to original concerns of Peirce.

What does exactly abduction and bi-abduction [CDOY11] consist in? Below, we propose two formulations: a general algebraic one (revealing a connection with unification) and a more specific one, sticking closely to both the proof system proposed in $\S 10.1$ and the paper of Calcagno et al. [CDOY11].

\subsection{Abduction and Bi-Abduction Algebraically}

Algebraically, one may think of the problem of abduction as follows: given

- a formal language $\mathscr{L}$ and a theory $T$ in $\mathscr{L}$ whose models all include as subreducts ordered monoids, with $\leq$ being the ordering (either primitive or term-definable) and · being the semigroup operation (again, either primitive or term-definable),

\footnotetext{
${ }^{17}$ Note that the denotation of pointer atoms is finite only if both PVar and Val are finite. Brotherston and Kanovich |BK14, $\S 10$ ] circumvent this by stating corresponding theorems in the heap-only setting, but given that the original result of Calcagno et al. CYO01] was proved for a store-and-heap model, a somewhat more general formulation would be desirable.

${ }^{18}$ Although when it comes to Peirce's own views, “[i]t is a common complaint that no coherent picture emerges from Peirce's writings on abduction. (Though perhaps this is not surprising, given that he worked on abduction throughout his career, which spanned a period of more than fifty years ....)" [Dou17].
} 
- two terms $h, c \in \mathscr{L}$ (called, respectively, the hypothesis and the conclusion),

- a set of potential antiframes (relative to $T, h$ and $c$ ) $\mathrm{Fr}^{-}(T, h, c) \subseteq \mathscr{L}$,

$$
\text { find } a \in F r^{-}(T, h, c) \text { s.t. } T \vdash h \cdot a \leq c \text {. }
$$

Whenever $\mathscr{L}$ and $T$ yield (either primitive or term-definable) left-residual $\backslash$ of $\cdot$, only the presence of $\mathrm{Fr}^{-}(T, h, c)$ prevents the problem from collapsing into triviality: otherwise, one could always take $a=h \backslash c$, and an even more dramatic trivialization would be possible whenever $T \vdash h \cdot \perp=\perp$. Furthermore, whereas traditional forms of abduction involve $\cdot$ being the additive multiplication $\wedge$, in the context of SL one is naturally interested in the spatial abduction, with "." being “ $*$ ”. Perhaps most importantly, however, from the point of view of concerns of SL, a more general (and symmetric) problem is of more interest. Calcagno et al. [CDOY11] baptised it bi-abduction.

Apart from taking as input data the same $\mathscr{L}, T \subseteq \mathscr{L}, h, c \in \mathscr{L}$ and $F r^{-}(T, h, c) \subseteq \mathscr{L}$, the problem of bi-abduction also requires potential frames (relative to $T, h$ and $c$ ) $\mathrm{Fr}^{+}(T, h, c) \subseteq \mathscr{L}$; needless to say, it can well happen that $F r^{-}(T, h, c)=F r^{+}(T, h, c)$. The problem is then to

$$
\text { find } a \in \mathrm{Fr}^{-}(T, h, c) \text { and } f \in F r^{+}(T, h, c) \text { s.t. } T \vdash h \cdot a \leq c \cdot f \text {. }
$$

Remark 11.1 Especially in the presence of semi-lattice connectives like $\wedge$, this general statement of biabduction can be reformulated as a special case of a restricted unification problem [Br86] (modulo theory). Namely, given fresh $x, y$, the challenge is to find a substitution $\sigma$ defined on $\{x, y\}$ (i.e., leaving other variables unchanged) s.t. $\sigma x \in \mathrm{Fr}^{-}(T, h, c), \sigma y \in F r^{+}(T, h, c)$ and $\sigma(h \cdot x \wedge c \cdot y)=\sigma(h \cdot x)$. We leave the exploration of this perspective for future investigation.

\subsection{Bi-Abduction in Separation Logic}

The above presentation of abduction and bi-abduction is much more general than the challenge of Calcagno et al. [CDOY11], which can be formulated concretely in terms of the proof system sketched in $\S 10.1$, given $H$ and $C$, find antiframe $\alpha$ and frame $\varphi$ s.t.

$$
H * \alpha \Rightarrow C * \varphi
$$

where not only $\alpha$ and $\varphi$, but also $H$ and $C$ themselves are symbolic heaps of the form $\exists \bar{v}$. $\Pi \wedge \Sigma, \Pi$ being a pure formula and $\Sigma$ being a spatial formula defined as follows:

$$
\begin{aligned}
\Pi, \Pi^{\prime}: & :=a_{1}=a_{2}\left|a_{1} \neq a_{2}\right| \top \mid \Pi \wedge \Pi^{\prime} \\
\Sigma, \Sigma^{\prime}:: & =a_{1} \mapsto a_{2}|e m p| \top \mid \Sigma * \Sigma^{\prime} .
\end{aligned}
$$

Furthermore, as already discussed in $\S 10.2$, there is no reason to insist on $a_{1}$ and $a_{2}$ being entirely arbitrary arithmetical expresssions. In fact, one often can restrict them to being elements of PVar, AVar plus a suitable collection of additional constants. On the other hand, as we also discussed in $\S 10.2$, one might often need a richer collection of spatial predicates, at the very least replacing $e \mapsto a$ with $e \mapsto_{\ell} \bar{a}$ and possibly more (cf., e.g., abstract predicates of Parkinson and Bierman [PB05]). A well-behaved class of similar formulas is the "Smallfoot fragment" (cf. $\S$ 12.2) as defined by Demri and Dieters [DD15a, $\S$ 4.3.1].

Just like in $\S 11.1$ abduction is a problem with the same input as bi-abduction, but the task is just to find antiframe $\alpha$ s.t. $H * \alpha \Rightarrow C$. Calcagno et al. [CDOY11] provide an analysis of minimality and termination of proof search for abduction in this setting, and a somewhat more sketchy one for bi-abduction, leaving a more throughout discussion of theoretical issues involved for future work. In $\S 12.2$ below, we are going to say a few more words about practical importance of (bi-)abduction for concrete tools.

\section{Applications and Later Developments}

In this section, we are going to briefly discuss applications, generalizations and developments which we cannot present in detail in this overview. 


\subsection{Competing Formalisms}

It would not be adequate to claim that SL has had no competitors to solve the problems plaguing Hoare reasoning about pointer programs presented at the beginning of $\S[9.2$. Bornat [Bor00] provides an overview of the state of the art exactly at the time when SL entered the scene.

Later, Kassios [Kas06] suggested another, object-oriented alternative in the form of the theory of $d y$ namic frames (concerning the name, recall Footnote 15). Soon afterwards, the theory of implicit dynamic frames [SJP09] rather successfully combined the insights of dynamic frames with those of SL. In particular, this has led to the continuing development of the tool VeriFast [Jac+11; Phi+14], whose core theory has been moreover formalized in the Coq proof assistant [JVP15]. One of most important features inherited by implicit dynamic frames from separation logic is the presence of $*$ in the assertion language.

\subsection{Tools}

VeriFast [Jac+11; JVP15; Phi+14] mentioned above is just one example of a recent, industrial-strength tool incorporating separation logic insights. But the story of such tools begins with Smallfoot [BCO06]. Its invention was preceded by investigation of decision procedures for fragments of the assertion language even better behaved than those appearing in $\S 10.2$ [BCO05a] (cf. also the discussion of the "Smallfoot fragment" in Demri and Deters [DD15a] and in $\S 11.2$ above) and symbolic execution in separation logic context $[\mathrm{BCO} 05 \mathrm{~b}]$. Another paper published at the same time which greatly contributed to subsequent popularity of SL and formalisms utilizing the frame rule was the work of Parkinson and Bierman [PB05] introducing abstract predicates.

Subsequently, the SL community produced more automated tools like SpaceInvader [Yan+08], SLAyer [BCI11] at Microsoft Research 19 (see also [BCO05b; DOY06] for underlying research on symbolic execution) and, especially, the static analyser Infer [CD11], presently developed at Facebook [Cal+15], but available open-source20. Infer crucially relies on frame inference and bi-abduction discussed in $\S 11$

So, a substructural logic is used in a tool that prevents thousands of bugs per month from reaching production in products used by over 1 billion people daily. [OHe]

Given the inherent computational limitations for fully algorithmic solutions, however, approaches based on proof assistants seem a natural alternative option, especially from an academic perspective. While there exists work on encoding separation logic, e.g., in Isabelle/HOL [Tue11], Coq seems the most common setting for such developments. Coq verification of Featherweight VeriFast [JVP15] illustrates that proof assistants may have a rôle to play even with fully automated tools. Another recent Coq-based line of work is a series of frameworks such as ModuRes [SBB15], Iris 2.0 [JKBD16], and MoSeL [Kre+18] with theoretical underpinnings in higher-order BI-hyperdoctrines [BBT07]. Finally, separation logic is also being gradually incorporated in Coq-based courses [Chl16; Pau16; Pie+18; Lit18].

\subsection{Concurrency and Algebraic Aspects}

The rich collection of models discussed in $\S \S 3$, 4 suggests that BI leads to more applications than reasoning about pointer programs in sequential separation logic. Of all such developments, we most regret not being able to devote more attention in this overview to concurrent separation logic. We can only refer the reader to a recent overview by Brookes and O'Hearn [BO16], which was written following the award of the 2016 Gödel Prize to both authors for their involvement in this formalism [Bro07; OHe07]. The very least we should say is that most tools and frameworks mentioned in $\S 12.2$ allow reasoning about concurrent programs. On the theoretical front, we only touched upon relevant issues in Remark 9.3

A development closely related to concurrent separation logic whose omission we particularly regret is concurrent Kleene algebra (CKA) [HMSW11; OPVH15]. And this is a good opportunity to finish by

19 https://github.com/Microsoft/SLAyer

${ }^{20}$ https://github.com/facebook/infer 
returning to the main algebraic theme of this overview. While equational features of Floyd-Hoare logics have been noticed and substantially used in monographs oriented towards category theory, like Manes and Arbib [MA86] or Bloom and Ésik [B93], a good reference for a more traditional algebraist is provided by Kozen [Koz00] showing how to encode Floyd-Hoare logics in Kleene algebra with tests (KAT; for an important predecessor see, e.g., Pratt [Pra76] discussing the relationship between Floyd-Hoare, Tarskian and modal semantics). It remains to be seen whether concurrent Kleene algebra with tests (CKAT, [Jip14; JM16]) is going to play a comparably important rôle. There is also an alternative algebraic approach to separation logic based on quantales [DHM11].

Acknowledgements We would like to thank: Hiroakira Ono, without whom both authors would not have met once upon a time in western Japan, there would have been no stimulus to write this overview, and many other things would not have happened; Nick Galatos and Kazushige Terui for suggesting the idea to write this overview, and for their patience and support during the very long write-up period; Nick, Peter O'Hearn, Revantha Ramanayake and Simon Docherty for their comments in the final stages of write-up, in Peter's case including the suggestion to add some material on bi-abduction $(\S 11$ and feedback regarding Infer and automated tools discussed in $\S 12.2$ Moreover, the second author wishes to thank: the family of the first author, in particular Julie Tapp, for hosting him for two weeks in April 2015, when the bulk of this paper was written; his project student Dominik Paulus for developing a convenient Coq formalization [Pau16], which proved helpful when working on $\S \S[9] 10$ and Erwin R. Catesbeiana, for displaying a tantalizing view on the empty heaplet.

\section{References}

[AKNSS96] H. Andrka,. Kurucz, I. Nmeti, I. Sain, and A. Simon. "Causes and remedies for undecidability in arrow logics and in multi-modal logics". In: Arrow Logic and Multi-Modal Logic. Stud. Logic Lang. Inform. CSLI Publications, 1996, pp. $63-100$

[AP01] P. A. Armeln and D. J. Pym. "Bunched logic programming". In: Proc. of IJCAR. Vol. 2083. LNCS. Springer, 2001, pp. 289-304.

[BJO04] F. Belardinelli, P. Jipsen, and H. Ono. "Algebraic aspects of cut elimination". Stud. Logica 77.2 (2004), pp. 209-240.

[Bel82] N. D. Belnap Jr. "Display logic". J. Philos. Log. 11.4 (1982), pp. 375-417.

[BCO05a] J. Berdine, C. Calcagno, and P. W. O'Hearn. "A decidable fragment of separation logic". In: Proc. of FSTTCS. 2005, pp. 97-109.

[BCO06] J. Berdine, C. Calcagno, and P. W. O'Hearn. "Smallfoot: modular automatic assertion checking with separation logic". In: Proc. of FMCO. 2006, pp. 115-137.

[BCO05b] J. Berdine, C. Calcagno, and P. W. O’Hearn. "Symbolic execution with separation logic". In: Proc. of APLAS. 2005, pp. 52-68.

[BCI11] J. Berdine, B. Cook, and S. Ishtiaq. "Slayer: memory safety for systems-level code". In: Proc. of CAV. 2011, pp. 178183 .

[BBT07] B. Biering, L. Birkedal, and N. Torp-Smith. "BI-hyperdoctrines, higher-order separation logic, and abstraction". ACM Trans. Program. Lang. Syst. 29.5 (2007).

[BP89] W. J. Blok and D. Pigozzi. Algebraizable Logics. Vol. 77. Memoirs AMS 396. AMS, 1989.

[BV02] W. J. Blok and C. J. Van Alten. "The finite embeddability property for residuated lattices, pocrims and BCK-algebras". Algebr. Univ. 48.3 (2002), pp. 253-271.

[B93] S. L. Bloom and Z. sik. Iteration Theories: The Equational Logic of Iterative Processes. Monogr. Theoret. Comput. Sci. EATCS Ser. Springer, 1993.

[BMR95] A. Borgida, J. Mylopoulos, and R. Reiter. "On the frame problem in procedure specifications". IEEE Trans. Softw. Eng. 21.10 (1995), pp. 785-798.

[Bor00] R. Bornat. "Proving pointer programs in Hoare logic". In: Proc. of MPC. 2000, pp. 102-126.

[BCY06] R. Bornat, C. Calcagno, and H. Yang. "Variables as resource in separation logic". In: Proc. of MFPS. Vol. 155. ENTCS. 2006, pp. 247-276.

[Bro07] S. Brookes. "A semantics for concurrent separation logic". Theor. Comput. Sci. 375.1-3 (2007), pp. 227-270.

[BO16] S. Brookes and P. W. O'Hearn. "Concurrent separation logic". ACM SIGLOG News 3.3 (2016), pp. 47-65.

[BC10] J. Brotherston and C. Calcagno. "Classical BI: its semantics and proof theory". LMCS 6.3 (2010).

[BK14] J. Brotherston and M. Kanovich. "Undecidability of propositional separation logic and its neighbours". J. ACM 61.2 (2014), 14:1-14:43.

[BK10] J. Brotherston and M. I. Kanovich. "Undecidability of propositional separation logic and its neighbours". In: Proc. of LiCS. 2010, pp. 130-139.

[BV14] J. Brotherston and J. Villard. "Parametric completeness for separation theories" (2014), pp. $453-464$.

[Br86] H.-J. Brckert. "Some relationships between unification, restricted unification, and matching". In: Proc. of CADE. 1986, pp. 514-524.

[Bur72] R. M. Burstall. "Some techniques for proving correctness of programs which alter data structures". In: Machine Intelligence. Vol. 7. Edinburgh, 1972, pp. 23-50.

[COY07] C. Calcagno, P. W. O'Hearn, and H. Yang. "Local action and abstract separation logic". In: Proc. of LiCS. 2007, pp. 366-378. 
[CDG10] C. Calcagno, T. Dinsdale-Young, and P. Gardner. "Adjunct elimination in context logic for trees". Inform. and Comput. 208.5 (2010), pp. 474-499.

[CD11] C. Calcagno and D. Distefano. "Infer: An automatic program verifier for memory safety of C programs". In: Proc. of NFM. 2011, pp. 459-465.

[CDOY11] C. Calcagno, D. Distefano, P. W. O'Hearn, and H. Yang. "Compositional shape analysis by means of bi-abduction". J. ACM 58.6 (2011), 26:1-26:66.

[CGZ05] C. Calcagno, P. Gardner, and U. Zarfaty. "Context logic and tree update". In: Proc. of POPL. 2005, pp. $271-282$.

[CGZ07] C. Calcagno, P. Gardner, and U. Zarfaty. "Context logic as modal logic: completeness and parametric inexpressivity". In: Proc. of POPL. 2007, pp. 123-134.

[CYO01] C. Calcagno, H. Yang, and P. W. O'Hearn. "Computability and complexity results for a spatial assertion language for data structures". In: Proc. of FSTTCS. 2001, pp. 108-119.

[Cal+15] C. Calcagno et al. "Moving fast with software verification". In: Proc. of NFM. 2015, pp. 3-11.

[CG04] L. Cardelli and G. Ghelli. "TQL: a query language for semistructured data based on the ambient logic". MSCS 14 (03 2004), pp. 285-327.

[CG00] L. Cardelli and A. D. Gordon. "Anytime, anywhere: modal logics for mobile ambients". In: Proc. of POPL. 2000, pp. 365-377.

[CFL10] B. ten Cate, G. Fontaine, and T. Litak. "Some modal aspects of XPath". J. Appl. Nonclassical Log. 20.3 (2010), pp. 139-171.

[CLM10] B. ten Cate, T. Litak, and M. Marx. "Complete axiomatizations for XPath fragments". J. Appl. Logic 8.2 (2010), pp. 153-172.

[CZ97] A. Chagrov and M. Zakharyaschev. Modal Logic. Oxford Logic Guides 35. Clarendon Press, 1997.

[Chl16] A. Chlipala. "Formal reasoning about programs". Online book and course material:http://adam. chlipala.net/frap/ 2016.

[CR17] A. Ciabattoni and R. Ramanayake. "Bunched hypersequent calculi for distributive substructural logics". In: Proc. of LPAR. Vol. 46. EPiC Series in Computing. 2017, pp. 417-434.

[CN10] P. Cintula and C. Noguera. "Implicational (semilinear) logics I: A new hierarchy". Arch. Math. Logic 49.4 (2010), pp. 417-446.

[Cla85] E. M. Clarke. "The characterization problem for Hoare logics". In: Proc. Of a Discussion Meeting of the Royal Society of London on Mathematical Logic and Programming Languages. 1985, pp. 89-106.

[CMP14] M. Collinson, K. McDonald, and D. Pym. "A substructural logic for layered graphs". J. Log. Comput. 24.4 (2014), pp. 953-988.

[Coo78] S. A. Cook. "Soundness and completeness of an axiom system for program verification". SIAM J. Comput. 7.1 (1978), pp. 70-90.

[Cuo+12] P. Cuoq et al. "Frama-c". In: Proc. of SEFM. 2012, pp. 233-247.

[DHM11] H.-H. Dang, P. Hfner, and B. Mller. "Algebraic separation logic". JLAMP 80.6 (2011), pp. $221-247$.

[DG03] B. A. Davey and J. C. Galati. “A coalgebraic view of Heyting duality". Stud. Logica 75 (2003), pp. $259-270$.

[DP02] B. A. Davey and H. A. Priestley. Introduction to lattices and order. Cambridge, 2002.

[DGG04] A. Dawar, P. Gardner, and G. Ghelli. "Adjunct elimination through games in static ambient logic". In: Proc. of FSTTCS. 2004, pp. 211-223.

[Day70] B. Day. "On closed categories of functors". In: Reports of the Midwest Category Seminar IV. Vol. 137. Lect. Notes Math. 1970, pp. 1-38.

[DD15a] S. Demri and M. Deters. "Separation logics and modalities: a survey". J. Appl. Nonclassical Log. (2015).

[DD15b] S. Demri and M. Deters. "Two-variable separation logic and its inner circle". ACM Trans. Comput. Logic 16.2 (2015), 15:1-15:36.

[DGLM14] S. Demri, D. Galmiche, D. Larchey-Wendling, and D. Mry. "Separation logic with one quantified variable". In: Proc. of CSR. 2014, pp. 125-138.

[DK00] M. Denecker and A. Kakas. "Special issue: abductive logic programming". J. Log. Prog. 44.1 (2000), pp. 1-4.

[DOY06] D. Distefano, P. W. O'Hearn, and H. Yang. "A local shape analysis based on separation logic". In: Proc. of TACAS. 2006, pp. 287-302.

[DP17a] S. Docherty and D. J. Pym. "Intuitionistic layered graph logic: Semantics and proof theory". CoRR (2017). arXiv: http://arxiv.org/abs/1710.03021

[DP17b] S. Docherty and D. J. Pym. "Stone-type dualities for separation logics". CoRR(2017). arXiv: http://arxiv . org/abs/1710.03021

[Dou17] I. Douven. "Abduction". In: The Stanford Encyclopedia of Philosophy. https://plato.stanford.edu/archives/sum2017/entr Metaphysics Research Lab, Stanford U., 2017.

[Dun75] J. M. Dunn. "Consecution formulation of positive R with co-tenability and t". In: Entailment: The Logic of Relevance and Necessity. Vol. 1. Princeton, 1975, pp. 381-391.

[EW97] U. Engberg and G. Winskel. "Completeness results for linear logic on Petri nets". Ann. Pure Appl. Logic 86.2 (1997), pp. 101-135.

[Flo67] R. W. Floyd. "Assigning meanings to programs". In: Mathematical Aspects of Computer Science. Vol. 19. Proc. Sympos. Appl. Math. AMS, 1967, pp. 19-32.

[Fonar] J. M. Font. "Abstract algebraic logic. An introductory chapter". In: Hiroakira Ono on Residuated Lattices and Substructural Logics. Outstanding Contributions to Logic. Springer, 2018 (to appear).

[FB94] D. J. Foulis and M. K. Bennett. "Effect algebras and unsharp quantum logics". Found. Phys. 24.10 (1994), pp. 13311352.

[Fre80] R. Freese. "Free modular lattices". Trans. Amer. Math. Soc. 261.1 (1980), pp. 81-91.

[Gal05] N. Galatos. "Minimal varieties of residuated lattices". Algebr. Univ. 52.2 (2005), pp. 215-239. 
[Gal00] N. Galatos. "Selected topics on residuated lattices". Qualifying paper, Department of Mathematics, Vanderbilt U. 2000 , p. 50.

[Gal02] N. Galatos. "The undecidability of the word problem for distributive residuated lattices". In: Developments in Mathematics 7. 2002, pp. 231-243.

[GJ17] N. Galatos and P. Jipsen. "Distributive residuated frames and generalized bunched implication algebras". Algebr. Univ. 78 (3 2017), pp. 303-336.

[GJ13] N. Galatos and P. Jipsen. "Residuated frames with applications to decidability". Trans. Amer. Math. Soc. 365.3 (2013), pp. 1219-1249.

[GJKO07] N. Galatos, P. Jipsen, T. Kowalski, and H. Ono. Residuated Lattices: An Algebraic Glimpse at Substructural Logics. Stud. Logic Found. Math. 151. 2007.

[GO10] N. Galatos and H. Ono. "Cut elimination and strong separation for substructural logics: An algebraic approach". Ann. Pure Appl. Logic 161.9 (2010), pp. 1097-1133.

[GR04] N. Galatos and J. G. Raftery. "Adding involution to residuated structures". Stud. Logica 77.2 (2004), pp. 181-207.

[GL06] D. Galmiche and D. Larchey-Wendling. "Expressivity properties of boolean BI through relational models". In: Proc. of FSTTCS. 2006, pp. 357-368.

[GMP05] D. Galmiche, D. Mry, and D. J. Pym. "The semantics of BI and resource tableaux". MSCS 15.6 (2005), pp. 10331088.

[HMT85] L. Henkin, J. Monk, and A. Tarski. Cylindric Algebras. Part II. Stud. Logic Found. Math. 115. North-Holland, 1985.

[Hoa69] C. A. R. Hoare. "An axiomatic basis for computer programming". Commun. ACM 12.10 (1969), pp. 576-580.

[HMSW11] T. Hoare, B. Mller, G. Struth, and I. Wehrman. "Concurrent Kleene algebra and its foundations". JLAMP 80.6 (2011), pp. 266-296.

[IO01] S. S. Ishtiaq and P. W. O'Hearn. "BI as an assertion language for mutable data structures". In: Proc. of POPL. 2001, pp. 14-26.

[JVP15] B. Jacobs, F. Vogels, and F. Piessens. "Featherweight VeriFast". LMCS 11 (3 2015).

[Jac+11] B. Jacobs et al. "VeriFast: A powerful, sound, predictable, fast verifier for C and Java". In: Proc. of NFM. 2011, pp. 41-55.

[Jan68] V. A. Jankov. "The construction of a sequence of strongly independent superintuitionistic propositional calculi". Soviet Mathematics Doklady 9 (1968).

[Jip92] P. Jipsen. "Computer-aided investigations of relation algebras". PhD thesis. Vanderbilt U., 1992.

[Jip14] P. Jipsen. "Concurrent Kleene algebra with tests". In: Proc. of RAMiCS. Vol. 8428. LNCS. 2014 , pp. 37-48.

[JM97] P. Jipsen and R. Maddux. "Nonrepresentable sequential algebras". Log. J. IGPL 5.4 (1997), pp. 565-574.

[JM16] P. Jipsen and M. A. Moshier. "Concurrent Kleene algebra with tests and branching automata". JLAMP 85.4 (2016), pp. 637-652.

[JT02] P. Jipsen and C. Tsinakis. “A survey of residuated lattices”. In: Developments in Mathematics 7. 2002 , pp. 19-56.

[JT93] B. Jnsson and C. Tsinakis. "Relation algebras as residuated boolean algebras". Algebr. Univ. 30.4 (1993), pp. 469-478.

[JKBD16] R. Jung, R. Krebbers, L. Birkedal, and D. Dreyer. "Higher-order ghost state". In: Proc. of ICFP. 2016, pp. 256-269.

[Kas06] I. T. Kassios. "Dynamic frames: support for framing, dependencies and sharing without restrictions". In: Proc. of FM. 2006, pp. 268-283.

[Kle99] T. Kleymann. "Hoare logic and auxiliary variables". Form. Asp. Comput. 11.5 (1999), pp. 541-566.

[Koz09] M. Kozak. "Distributive full Lambek calculus has the finite model property". Stud. Logica 91.2 (2009), pp. 201-216.

[Koz00] D. Kozen. "On Hoare logic and Kleene algebra with tests". ACM Trans. Comput. Logic 1.1 (2000), pp. 60-76.

[Kre+18] R. Krebbers et al. "MoSeL: A general, extensible modal framework for interactive proofs in separation logic". Proc. ACM Program. Lang. 2.ICFP (2018), 77:1-77:30.

[KNSS95] . Kurucz, I. Nmeti, I. Sain, and A. Simon. "Decidable and undecidable logics with a binary modality". JoLLI 4.3 (1995), pp. 191-206.

[LG09] D. Larchey-Wendling and D. Galmiche. "Exploring the relation between Intuitionistic BI and Boolean BI: An unexpected embedding". MSCS 19.3 (2009), pp. 435-500.

[LG13] D. Larchey-Wendling and D. Galmiche. "Nondeterministic phase semantics and the undecidability of boolean BI". ACM Trans. Comput. Logic 14.1 (2013), 6:1-6:41.

[LG10] D. Larchey-Wendling and D. Galmiche. "The undecidability of boolean BI through phase semantics". In: Proc. of LiCS. 2010, pp. 140-149.

[Lei10] K. R. M. Leino. "Dafny: an automatic program verifier for functional correctness". In: Proc. of LPAR. Springer Berlin Heidelberg, 2010, pp. 348-370.

[Lip74] L. Lipshitz. "The undecidability of the word problems for projective geometries and modular lattices". Trans. Amer Math. Soc. 193 (1974), pp. 171-180.

[Lit18] T. Litak. Lecture material of SemProg 2018 @FAU: Our fork of [Pie+18]. URL/DOI: http://dx.doi . org/10.13140/RG.2.2.10542 2013-2018.

[LPR17] T. Litak, M. Polzer, and U. Rabenstein. "Negative translations and normal modality". In: Proc. of FSCD. Vol. 84. LIPIcs. 2017, 27:1-27:18.

[Loz04] . Lozes. "Adjuncts elimination in the static ambient logic". In: Proc. of the 10th International Workshop on Expressiveness in Concurrency. Vol. 96. ENTCS. 2004, pp. 51-72.

[Mad06] R. D. Maddux. Relation Algebras. Stud. Logic Found. Math. 150. 2006.

[MA86] E. G. Manes and M. A. Arbib. Algebraic approaches to program semantics. The AKM Series in Theoretical CS. 1986.

[MPM96] M. Marx, L. Plos, and M. Masuch (eds). Arrow Logic and Multi-Modal Logic. Stud. Logic Lang. Inform. CSLI Publications, 1996.

[MH69] J. McCarthy and P. J. Hayes. "Some philosophical problems from the standpoint of artificial intelligence". In: Machine Intelligence 4. Edinburgh, 1969, pp. 463-502. 
[MT46] J. C. C. McKinsey and A. Tarski. "On closed elements in closure algebras". Annals of Mathematics 47.1 (1946), pp. $122-162$.

[Mey97] B. Meyer. Object-Oriented Software Construction. 2nd. Prentice-Hall, Inc., 1997.

[Mik96] S. Mikuls. "Complete calculus for conjugated arrow logic". In: Arrow Logic and Multi-Modal Logic. Stud. Logic Lang. Inform. CSLI Publications, 1996, pp. 125-139.

[Min76] G. E. Mints. "Cut-elimination theorem for relevant logics". Journal of Soviet Mathematics 6.4 (1976), pp. 422-428.

[Neu60] J. von Neumann. Continuous Geometry. Princeton, 1960.

[OHe12] P. W. O'Hearn. "A primer on separation logic (and automatic program verification and analysis)". In: Software Safety and Security. Vol. 33. NATO SPS Series D. 2012, pp. 286-318.

[OHe] P. W. O'Hearn. "Email to T. Litak and P. Jipsen, 29 August 2017".

[OHe99] P. W. O'Hearn. "Resource interpretations, bunched implications and the $\alpha \lambda$-calculus (preliminary version)". In: Proc. of TLCA. Vol. 1581. LNCS. 1999, pp. 258-279.

[OHe07] P. W. O'Hearn. "Resources, concurrency, and local reasoning". Theor. Comput. Sci. 375.1 (2007), pp. $271-307$.

[OPVH15] P. W. O'Hearn, R. L. Petersen, J. Villard, and A. Hussain. "On the relation between Concurrent Separation Logic and Concurrent Kleene Algebra”. JLAMP 84.3 (2015), pp. 285-302.

[OP99] P. W. O'Hearn and D. J. Pym. "The logic of bunched implications". 5.2 (1999), pp. $215-244$.

[OYR09] P. W. O'Hearn, H. Yang, and J. C. Reynolds. "Separation and information hiding". ACM Trans. Program. Lang. Syst. 31.3 (2009), pp. 1-50.

[ORY01] P. O'Hearn, J. Reynolds, and H. Yang. "Local reasoning about programs that alter data structures". In: Proc. of CSL. 2001, pp. 1-19.

[PBC06] M. Parkinson, R. Bornat, and C. Calcagno. "Variables as resource in Hoare logics". In: Proc. of LiCS. 2006, pp. 137146.

[PB05] M. Parkinson and G. Bierman. "Separation logic and abstraction". In: Proc. of POPL. 2005, pp. 247-258.

[Pau93] G. Paul. "Approaches to abductive reasoning: an overview". Artif. Intell. Rev. 7.2 (1993), pp. $109-152$.

[Pau16] D. Paulus. ImpDynamic: Dynamic memory allocation and separation logic in the style of Software Foundations. A Coq formalization supervised by T. Litak, part of plf18 volume of [Lit18]. 2016.

[Pei58] C. S. Peirce. Collected Papers of Charles Sanders Peirce, edited by C. Hartshorne, P. Weiss, and A. Burks. Harvard, 1931-1958.

[Phi+14] P. Philippaerts et al. "Software verification with VeriFast: industrial case studies". Sci. Comput. Programming 82 (2014), pp. 77-97.

[Pie+18] B. C. Pierce et al. Software Foundations. Version 5.6 (25 Aug 2018, Coq 8.8.0). Electronic textbook, http: / / www . cis . upenn . edu / bcp 2018.

[Pra91] V. Pratt. "Action logic and pure induction". In: Logics in AI. Vol. 478. LNCS. 1991, pp. 97-120.

[Pra76] V. R. Pratt. "Semantical consideration on Floyd-Hoare logic". In: Proc. of the 17th SFCS. 1976, pp. 109-121.

[Pym02] D. Pym. The Semantics and Proof Theory of the Logic of Bunched Implications. Vol. 26. Applied Logic Series. Kluwer Academic Publishers, 2002.

[Pym99] D. J. Pym. "On bunched predicate logic". In: Proc. of LiCS. 1999, pp. 183-192.

[POY04] D. J. Pym, P. W. O’Hearn, and H. Yang. "Possible worlds and resources: the semantics of BI". Theor. Comput. Sci. 315.1 (2004), pp. 257-305.

[Rafar] J. Raftery. "Universal algebraic methods for non-classical logics". In: Hiroakira Ono on Residuated Lattices and Substructural Logics. Outstanding Contributions to Logic. Springer, 2018 (to appear).

[Ram16] R. Ramanayake. "A purely syntactic proof of decidability for BI". CoRR (2016). arXiv: http://arxiv. org/abs/1609.05847

[Ras74] H. Rasiowa. An Algebraic Approach to Non-classical Logics. Vol. 78. Stud. Logic Found. Math. North-Holland, 1974.

[Rea88] S. Read. Relevant Logic: A Philosophical Examination of Inference. B. Blackwell, 1988.

[Rey00] J. C. Reynolds. "Intuitionistic reasoning about shared mutable data structure". In: Millennial Perspectives in Computer Science. Palgrave, 2000, pp. 303-321.

[Rey02] J. C. Reynolds. "Separation Logic: A logic for shared mutable data structures". In: Proc. of LiCS. 2002, pp. 55-74.

[SBB15] F. Sieczkowski, A. Bizjak, and L. Birkedal. "ModuRes: A Coq library for modular reasoning about concurrent higherorder imperative programming languages". In: Proc. of ITP. 2015, pp. 375-390.

[SJP09] J. Smans, B. Jacobs, and F. Piessens. "Implicit dynamic frames: combining dynamic frames and separation logic". In: Proc. of ECOOP. 2009, pp. 148-172.

[Tar41] A. Tarski. "On the calculus of relations". J. Symb. Log. 6.3 (1941), pp. 73-89.

[TS96] A. Troelstra and H. Schwichtenberg. Basic Proof Theory. Cambridge, 1996.

[Tue11] T. Tuerk. A separation logic framework for HOL. Tech. rep. UCAM-CL-TR-799. U. of Cambridge, Computer Laboratory, 2011.

[Urq95] A. Urquhart. "Decision problems for distributive lattice-ordered semigroups". Algebr. Univ. 33.3 (1995), pp. 399-418.

[Urq96] A. Urquhart. "Duality for algebras of relevant logics". Stud. Logica 56.1-2 (1996). Special issue on Priestley duality, pp. 263-276.

[Urq72] A. Urquhart. "Semantics for relevant logics". J. Symb. Log. 37.1 (1972), pp. 159-169.

[Urq84] A. Urquhart. "The undecidability of entailment and relevant implication". J. Symb. Log. 49.4 (1984), pp. 1059-1073.

[Win93] G. Winskel. The Formal Semantics of Programming Languages: An Introduction. MIT Press, 1993.

[Yan01] H. Yang. "Local Reasoning for Stateful Programs". PhD thesis. U. of Illinois, 2001.

[Yan+08] H. Yang et al. "Scalable shape analysis for systems code". In: Proc. of CAV. 2008, pp. 385-398. 